\title{
Hērōs ed hērōikai timai nel mondo greco ellenistico e imperiale: semantica e prassi, attori e contesti
}

Hērōs and hērōikai timai in the Greek Hellenistic and Imperial World:

Semantics and Practice, Agents and Contexts

\section{Stefano G. Caneva e Alessandra Coppola}

\section{OpenEdition}

\section{Journals}

\section{Edizione digitale}

URL: https://journals.openedition.org/mythos/2011

DOI: 10.4000/mythos.2011

ISSN: 2037-7746

\section{Editore}

Salvatore Sciascia Editore

\section{Notizia bibliografica digitale}

Stefano G. Caneva e Alessandra Coppola, «Hērōs ed hērōikai timai nel mondo greco ellenistico e imperiale: semantica e prassi, attori e contesti», Mythos [Online], 14 | 2020, online dal 31 décembre 2020, consultato il 05 octobre 2021. URL: http://journals.openedition.org/mythos/2011 ; DOI: https:// doi.org/10.4000/mythos.2011

Questo documento è stato generato automaticamente il 5 octobre 2021.

Mythos 


\section{Hērōs ed hērōikai timai nel mondo greco ellenistico e imperiale: semantica e prassi, attori e contesti ${ }^{1}$}

Hērōs and hērōikai timai in the Greek Hellenistic and Imperial World: Semantics and Practice, Agents and Contexts

Stefano G. Caneva e Alessandra Coppola

\section{Alla ricerca dell'hērōs nel mondo greco post-classico: fonti, metodi di ricerca e problemi}

1 Questo studio intende offrire un contributo alla riflessione sull'evoluzione della sfera semantica della parola n̆ $\rho \omega \varsigma$ e dei suoi derivati in età ellenistica e imperiale, attraverso l'analisi delle attestazioni epigrafiche e letterarie e con una attenzione particolare per la documentazione relativa alla concessione e pratica di onori cultuali per defunti, in contesto pubblico o privato $^{2}$. Il nostro approccio sarà sincronico e diacronico: discuteremo cioè le varie declinazioni della sfera semantica di n̆ $\rho \omega \varsigma$ da un punto di vista di sistema socio-linguistico, esplorandone i vari contesti di utilizzo, con le loro differenze legate alla distribuzione regionale e al tipo di attore, testo e supporto; cercheremo d'altro canto di mettere in risalto le dinamiche e i mutamenti che tale sistema subì nel corso del tempo.

2 L'analisi diacronica dei rapporti fra rappresentazione religiosa, prassi onorifica e vocabolario funebre, implicati dalla categoria eroica, rende necessaria una riflessione interdisciplinare, che tenga conto insieme di fattori linguistici, religiosi, giuridici e socio-culturali. Si pone innanzitutto la questione del rapporto, all'interno di una comunità, fra la pratica di onorare personaggi storici come hērōes e l'immagine che tale comunità ha degli eroi tradizionali e del loro culto. La critica recente ha sottolineato come la prassi ellenistica e imperiale dell'eroizzazione conviva con un rinnovato vigore nella frequentazione e monumentalizzazione di luoghi di culto degli eroi del mito ${ }^{3}$. 
D'altro canto, fin dall'età arcaica, il culto per vecchi e nuovi eroi svolse un ruolo importante nelle strategie sociali e nella promozione dell'identità politico-religiosa delle comunità che lo praticavano ${ }^{4}$. Ci si può dunque chiedere in che misura i nuovi hêrōes ellenistici abbiano contribuito alla costituzione di legami di gruppo in un quadro storico profondamente rinnovato e caratterizzato da forti differenze interne, come è quello del Mediterraneo orientale dopo Alessandro. Non potremo poi astenerci dal discutere l'annosa questione se la crescente diffusione del termine ク̆ vocabolario funebre di età tardo-ellenistica e imperiale debba essere intesa come un segno di banalizzazione di questa categoria religiosa, come a lungo si è ritenuto ${ }^{5}$, o se, al contrario, tale pratica onorifica testimoni piuttosto la vitalità di tale categoria e la sua capacità di rispondere alle esigenze dell'immaginario religioso di fasce via via più ampie della popolazione ${ }^{6}$. Infine, è necessario fin d'ora sottolineare l'importanza di distinguere fra documenti nei quali il riferimento alla sfera semantica di n̆ $\rho \omega \varsigma$ si accompagna a una documentata specificità rituale, e altri in cui la categoria eroica sembra piuttosto evocata a un livello discorsivo ${ }^{7}$. All'interno di questa seconda categoria proponiamo di distinguere tre funzioni principali, sulle quali torneremo nel nostro contributo: una retorico-onorifica ${ }^{8}$, una giuridico-sepolcrale ${ }^{9}$ e una legata all'immaginario relativo al destino ultraterreno del defunto ${ }^{10}$. Come si vedrà, tale distinzione non è sempre di facile applicazione e in molti casi le fonti suggeriscono una sovrapposizione fra le varie funzioni qui evocate; tuttavia l'ipotesi che la categoria eroica possa essere evocata anche in contesti differenti dalla sfera dei rituali funebri eccezionali per figure storiche o mitiche risulterà utile per descrivere e comprendere le innovazioni del periodo tardo-ellenistico e suprattutto imperiale.

3 Lungi da una pretesa di esaustività, lo scopo del presente studio è quello di condividere una serie di prospettive di ricerca aperte da due progetti paralleli incontratisi negli anni scorsi all'Università di Padova: lo spoglio della documentazione epigrafica concernente la sfera semantica di n̆ $\rho \omega \varsigma$, diretto da A. Coppola ${ }^{11}$, e il progetto Marie Curie Piscopia di S. Caneva, The Practicalities of Hellenistic Ruler Cults (PHRC), dedicato alla dimensione rituale degli onori cultuali per i capi politici dell'età ellenistica ${ }^{12}$.

4 La documentazione presa in esame abbraccia i secoli che intercorrono fra l'ascesa dei Diadochi (fine IV sec. a.C.) e la media età imperiale (II/III sec. d.C.). Si tratta di una "lunga età ellenistica" sempre più spesso percepita dagli specialisti come necessaria a rendere conto delle durature dinamiche socio-culturali del Mediterraneo orientale: una continuità che si impone al di là della troppo puntuale cesura militare di Azio e della svolta politica imposta dalla formazione del Principato, che solo parzialmente modificò le tendenze storiche di lungo periodo nelle regioni orientali già da tempo integrate nell'imperium ${ }^{13}$. L'ampio taglio cronologico che abbiamo scelto costituisce una soluzione metodologica essenziale per apprezzare appieno il dispiegarsi di alcune tendenze fondamentali.

5 Nella prima parte della nostra comunicazione, presenteremo un bilancio dell'analisi sistematica condotta sulle occorrenze epigrafiche della parola n̆ $\rho \omega \varsigma$, in tutte le sue declinazioni e forme grafiche, al maschile e al femmininile, e della famiglia di parole ad essa collegate, come $\dot{\eta} \rho \omega \tilde{o} o v, \dot{\eta} \rho \omega \ddot{o} \sigma \bar{\eta} \varsigma$ e $\dot{\alpha} \varphi \eta \rho \omega \hat{i} \zeta \omega$. Passeremo quindi dalla discussione lessicale a un tentativo di analisi sociologica, nella quale maggiore attenzione sarà riconosciuta ai differenti attori coinvolti e ai contesti nei quali la famiglia lessicale di ท̆ $\rho \omega \varsigma$ è impiegata nel quadro di pratiche onorifiche. Sebbene i risultati presentati in questa occasione debbano essere considerati come parziali, essi ci sembrano offrire le 
premesse per una messa a punto di alcune problematiche centrali legate agli sviluppi della categoria eroica nella religione e più ampiamente nella vita sociale delle comunità greche o ellenizzate del periodo ellenistico e imperiale. A questa discussione generale saranno dedicate le nostre considerazioni conclusive.

\section{II nome ňpws}

6 La schedatura condotta all'Università di Padova sulla parola ク̆

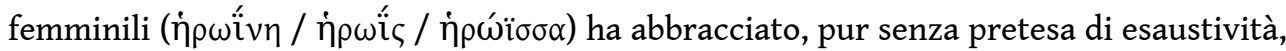
l'intero arco cronologico dell'Antichità greca dal periodo arcaico all'età imperiale. Nei casi documentati per il periodo che qui ci interessa, n̆ $\rho \omega \varsigma$ presenta contesti di utilizzo differenti: può fare riferimento agli eroi del mito panellenico (con consistenza minoritaria), oppure comparire nella denominazione di figure eroiche locali ${ }^{14}$; ancora, ed è il contesto che più direttamente ci riguarda, può riferirsi a un personaggio storico destinatario di onori che lo distinguono dagli altri mortali su un piano religioso e/o sociale. Pressoché nella totalità della documentazione, le iniziative onorifiche marcate dalla sfera semantica di n̆ $\rho \omega \varsigma$ si lasciano senza dubbio comprendere come funebri. Come vedremo, il contenuto di tali onori è estremamente variabile e richiede di essere analizzato caso per caso ${ }^{15}$.

7 La parola n̆ $\rho \omega \varsigma$ è attestata nelle iscrizioni ellenistiche e imperiali in tutti i casi della declinazione ed è possibile, per ogni caso, riassumere alcune forme di utilizzo ricorrenti. Al nominativo o vocativo, n̆ $\rho \omega \varsigma$ accompagna di solito come apposizione il nome proprio di un defunto, spesso in combinazione con la formula di commiato

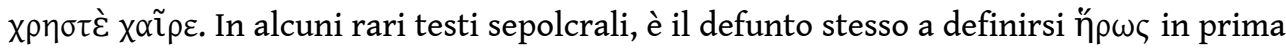
persona ${ }^{16}$. Un aggettivo può qualificare il nome ň $\rho \omega \varsigma$, come ad esempio $\alpha \gamma \alpha \theta$ ó, le cui ricorrenze risultano particolarmente comuni nella documentazione proveniente dalla Magna Grecia e dalla Sicilia. Al genitivo singolare, ท̆ defunto, si riferisce comunemente al proprietario di una tomba o completa un sintagma indicante un figlio del defunto eroizzato o un responsabile del culto. Fra le occorrenze

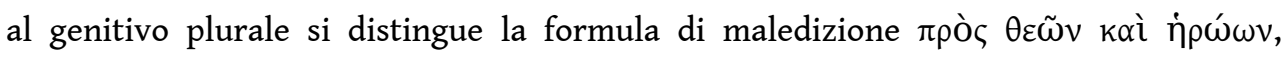
attestata in vari atti di fondazione cultuale e diretta contro chi osasse arrecare danno a un luogo sacro ${ }^{17}$. Un uso particolare del genitivo plurale si segnala nella funzione partitiva che accompagna un superlativo relativo: in questo caso, il testo stabilisce un paragone fra la persona onorata e l'insieme degli eroi ${ }^{18}$.

8 Un caso degno di nota, attestato in vari documenti civici provenienti dall'Asia Minore meridionale di età imperiale, è costituito dalla formula di datazione con غ̇ंí + la carica pubblica eponima e il nome proprio del titolare della carica seguito dall'apposizione

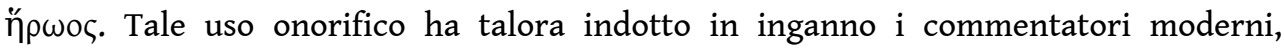
suggerendo che l'onorato fosse già riconosciuto come ň $\rho \omega \varsigma$ in vita. Come già dimostrato da Robert, tuttavia, la formula rimanda alla possibilità, da parte di un membro dell'élite civica, di istituire un fondo destinato a garantire l'esercizio continuativo della carica eponima non solo in vita, ma anche, virtualmente, post mortem $^{19}$.

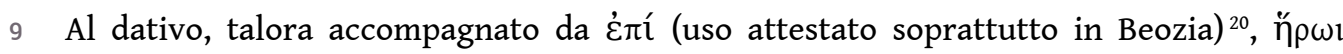
indica il destinatario di una dedica. Può comparire da solo o insieme al nome proprio dell'onorando, nel qual caso svolge funzione appositiva. Di particolare interesse per la 
documentazione epigrafica è l'assenza pressoché totale della formula $\dot{\omega} \varsigma$ (o più

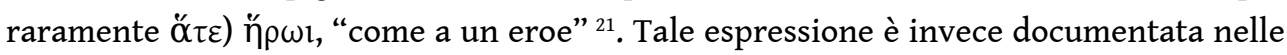
fonti letterarie già in età classica, in frasi indicanti l'attribuzione di onori a un tale "come a un eroe", pur diventando più rara, anche in letteratura, proprio in età ellenistica $^{22}$. Se quindi alcuni passi letterari, specialmente pre-ellenistici, pongono l'accento sulla natura secondaria degli onori eroici per umani, rispetto a quelli per gli eroi mitici, che ne sono il modello, nella documentazione ellenistica (e in particolare in quella epigrafica) l'onorato appare direttamente, e senza bisogno di un confronto con il modello tradizionale, come il destinatario di un atto rituale indirizzato a un eroe. Lo stesso verbo che esprime l'atto d'omaggio è spesso omesso, né possiamo trovare traccia, al di là del raro verbo $\dot{\alpha} \varphi \eta \rho \omega \hat{\zeta} \zeta \omega$ su cui torneremo, di espressioni che attirino l'attenzione in modo sistematico sull'atto con il quale si stabilisce o proclama lo statuto eroico dell'onorato.

Al plurale, il dativo ń $\rho \omega \sigma$ si riferisce alla totalità degli eroi in quanto destinatari di un'azione rituale. In questo caso, gli eroi non sono menzionati da soli, ma compaiono in una formula che li affianca agli dei, per indicare che l'offerta è dedicata a tutte le potenze divine ed eroiche onorate dal dedicante o dalla comunità ${ }^{23}$. In contesti di contatto con la cultura romana - in Occidente o nel caso di Romani o Italici in Oriente l'espressione $\theta \varepsilon$ coi costituisce un equivalente della formula latina Dis Manibus ${ }^{24}$. Infine, le attestazioni dell'accusativo ท̌ $\rho \omega$ appaiono in genere in dipendenza da un verbo, spesso sottinteso,

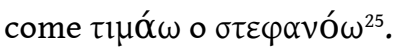

11 Una concentrazione particolare del nome n̆ $\omega \varsigma$ si segnala nelle iscrizioni della Grecia centrale, delle isolee egee e dell'Asia Minore occidentale ${ }^{26}$. Applicata a una prospettiva geografica, l'analisi morfologica permette inoltre di identificare alcune differenze nell'usus. Ad esempio, la formula al nominativo e al vocativo è molto frequente in Grecia centrale e settentrionale, mentre le formule al genitivo sono molto più diffuse in Asia Minore e nelle isole. Sul piano delle differenze di genere, le occorrenze femminili confermano nella loro distribuzione la concentrazione della categoria eroica nelle isole e in Asia Minore (in particolare nelle forme $\dot{\eta} \rho \omega \hat{i} v \eta$ e $\dot{\eta} \rho \omega \hat{i} \varsigma$ ) e in Grecia centrale (principalmente $\dot{\eta} \rho \omega ́ i ̈ \sigma \sigma \alpha)$. Il totale femminile è tuttavia decisamente inferiore a quello maschile, con un rapporto di quasi 1 a $10^{27}$.

\section{La categoria di ñ $\rho \omega \varsigma$ fra eroi mitici e nuovi eroi}

La documentazione concernente la pratica di culti eroici nelle comunità greche o ellenizzate di età ellenistica e imperiale getta luce sia sulla sopravvivenza di culti preesistenti (legati al patrimonio mitico panellenico o a realtà cultuali locali), sia sulla fioritura di culti per eroi non documentati nelle fonti di età classica. Fra questi, è possibile distinguere tra figure appartenenti al novero degli eroi dell'epopea e altre la cui denominazione comprende la parola ท̆ $\rho \omega \varsigma$ accompagnata da un altro nome in funzione appositiva. In questi casi, la seconda componente del nome caratterizza il

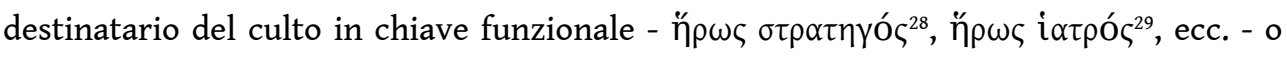
più raramente toponimica. Tale fioritura di culti eroici nuovi (o comunque non noti da fonti precedenti) conferma la vitalità di tale aspetto della religione greca nelle sue fasi post-classiche. 
Il nuovo e significativo successo degli onori accordati a defunti, il cui statuto è semanticamente marcato dalla famiglia lessicale di avviso come un ulteriore segnale della vivacità di tale categoria religiosa nel mondo greco ellenistico-imperiale. Tale diffusione di onori funebri eroizzanti solleva la questione di come gli antichi percepissero il rapporto fra vecchi e nuovi hērōes. Di particolare interesse in questa direzione sono alcune iscrizioni sepolcrali che stabiliscono un nesso esplicito fra il defunto onorato e gli eroi del mito. La convinzione che il defunto eroizzato venga a collocarsi sullo stesso piano degli hērōes antichi è espressa attraverso rimandi mitologici che sottolineano la prossimità in termini di onomastica, provenienza geografica o, più genericamente, di eccellenza $(\dot{\alpha} \rho \varepsilon \tau)^{30}{ }^{30}$. Nel caso di onorandi appartenenti a famiglie nobili, la continuità fra defunti ed eroi del mito può essere esplicitata in una chiave propriamente genealogica. Il poema commissionato nel 161 d.C. da Erode Attico per la morte della moglie Regilla è rivelatore di tale processo ${ }^{31}$. Il richiamo, da parte delle élites civiche, a un mondo mitologico presentato come passato familiare è del resto una tendenza comune nelle poleis greche di età imperiale, dove il classicismo dominante offre alle élites, attraverso i richiami genealogici al mito panellenico, un'occasione per affermare la propria posizione di prestigio nel mondo globale dell'impero romano ${ }^{32}$.

\section{Il monumento funebre come ท์}

La maggior parte della documentazione epigrafica del termine $\dot{\eta} \rho \tilde{\omega} o v$ proviene dall'Asia Minore ${ }^{33}$. Il classico studio di Kubińska sui monumenti funerari attraverso le iscrizioni greche micro-asiatiche, insieme alla recente panoramica di Mikkola sugli hērōa nelle fonti epigrafiche e letterarie (Ateneo, Strabone, Pausania, Plutarco), hanno affinato la nostra comprensione della terminologia relativa alle tombe di eroi mitici e di persone storiche "eroizzate" ${ }^{34}$. Tali studi hanno messo in luce differenze d'uso a livello sia regionale sia d'autore, ma anche alcune tendenze generali di fondo. Così, da un lato l'interesse per le tombe di eroi storici varia fra scrittore e scrittore (Plutarco si limita a ricordare l'hērōon di Arato, mentre Pausania menziona sette hērōa di figure storiche nel Peoloponneso ${ }^{35}$ ); d'altro canto la fluidità delle scelte lessicali di Pausania - che usa

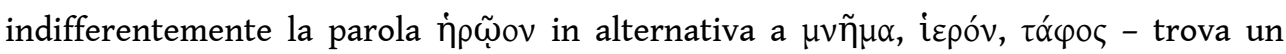
confronto diretto nell'altrettanto fluido vocabolario della coeva documentazione epigrafica.

Rispetto al taphos, che fa riferimento alla funzione della tomba in quanto luogo di sepoltura, hērōon sembra piuttosto sottolineare la sacralità dell'edificio, inviolabile ed inalienabile ${ }^{36}$ perché inteso a commemorare il defunto eroizzato e a ospitare gli eventuali rituali celebrati in suo onore ${ }^{37}$. Il verbo consueto usato per indicare l'erezione

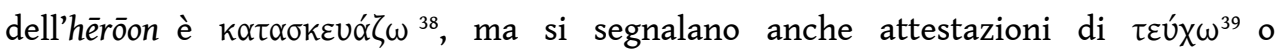
semplicemente di $\pi 01 \varepsilon \omega^{40}$. I testi iscritti sul monumento funebre presentano spesso prescrizioni contro le violazioni della tomba e soprattutto contro l'uso illecito del sepolcro, con un'ampia documentazione di multe comminate ai trasgressori da parte delle istituzioni pubbliche o dell'associazione incaricata della tutela del momumento. In alcuni casi, l'ammenda pecuniaria può essere accompagnata da una maledizione contro chi intendesse violare o usare illecitamente il monumento funebre. 


\section{Il verbo $\alpha \dot{\varphi} \eta \rho \omega i ̂ ́ \zeta \omega$}

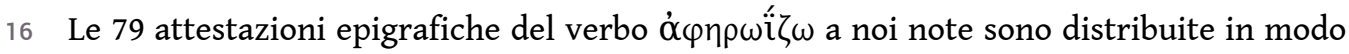
molto disomogeneo dal punto di vista sia cronologico sia geografico ${ }^{41}$. A fronte di 2 occorrenze ellenistiche a Creta e in Attica, alle quali va aggiunta la sola attestazione del sostantivo derivato $\dot{\alpha} \varphi \eta \rho \omega \ddot{\sigma} \sigma$ ó $\varsigma$ ad Aigiale di Amorgo, il resto della documentazione appartiene al periodo imperiale, fra il I e il III sec. d.C., e presenta una ripartizione decisamente sbilanciata a favore di Thera, dove si concentrano più di tre quarti dell'intero dossier, testimoniando così un vero e proprio usus epigrafico che coinvolge anche la vicina Anaphe ${ }^{42}$.

17 Al quadro offerto dalla distribuzione della documentazione corrisponde anche una differenzazione per quel che riguarda il contesto di utilizzo del verbo. Se infatti i tre documenti ellenistici impiegano il verbo e il suo sostantivo derivato in testi che evocano onori rituali per defunti eroizzati, le fonti di età imperiale non presentano alcun dettaglio di carattere rituale ma si inquadrano chiaramente in due tipologie di fonti: testi che fanno riferimento generico all'eroizzazione di un defunto da parte della città (documenti pubblici) o dei familiari (documenti privati); documenti giuridici concernenti la protezione della tomba di un individuo o di una famiglia.

Un epigramma funebre di I sec. a.C. da Itanos (Creta orientale) ricorda tre giovani fratelli morti ed eroizzati dalla città in segno di ringraziamento verso l'attività evergetica del padre di questi. Il testo evoca un decreto civico che ha sancito lo statuto

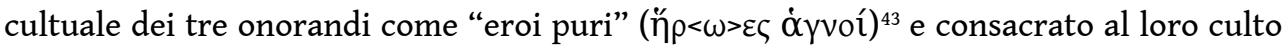

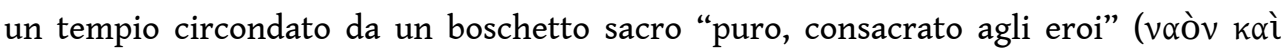

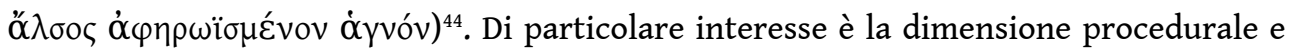
istituzionale che l'azione espressa dal verbo $\dot{\alpha} \varphi \eta \rho \omega \hat{i} \zeta \omega$ prende in questo testo: l'eroizzazione dei giovani e la consacrazione di spazi per il loro culto eroico dipendono esplicitamente da una decisione delle istituzioni civiche con valore legale. La stessa dimensione legale si segnala in un decreto di Aigiale concernente la fondazione cultuale stabilita da Kritolaos per il proprio figlio Aleximachos, morto durante il periodo di addestramento efebico (IG XII 7 515; II sec. a.C.) ${ }^{45}$. Il testo che introduce e regola la fondazione, un lungo documento esposto all'interno del ginnasio, rievoca l'attribuzione dello statuto eroico al defunto Aleximachos in termini chiaramente appartenenti alla sfera delle procedure legali della città ${ }^{46}$. Il documento elenca quindi il funzionamento delle cerimonie in onore di Aleximachos e descrive nel dettaglio il meccanismo di gestione del fondo necessario al loro finanziamento ${ }^{47}$.

Nell'ultimo testo ellenistico a noi noto (IG II $^{2} 1326 ; 176 / 5$ a.C.), il rapporto fra élite e istituzioni civiche lascia il posto a un contesto sociale interamente inscritto nel quadro privato di una famiglia aristocratica del Pireo e del gruppo chiuso delle sue frequentazioni, riunite in una associazione dedita al culto di Dioniso. Gli orgeones di Dioniso decretano che Dionysios, il presidente e benefattore dell'associazione cultuale, sia eroizzato e una sua statua sia dedicata presso il dio, nel tempio, come già era accaduto per suo padre, affinché resti splendido ricordo di lui per sempre ${ }^{48}$.

In età imperiale, l'impiego legale e procedurale del verbo $\alpha \dot{\alpha} p \rho \omega i ̂ ́ \zeta \omega$ continua a costituire un tratto evidente della documentazione, in particolare nei testi concernenti la tutela del monumento funebre contro il rischio di spoliazioni o di usi illeciti da parte di persone altre da quelle con cui il proprietario del monumento ha deciso di 
condividere il diritto di sepoltura. Attraverso tale tipologia di documenti, ben attestata in particolare in Asia Minore e in Tracia/Mesia inferiore, il proprietario di una tomba regola l'accesso al diritto di sepoltura nel proprio monumento funebre e istituisce una multa per i trasgressori, talora rafforzando il controllo umano, affidato a una istituzione della città, con una maledizione contro chi intendesse comunque rimuovere, vendere o riutilizzare la tomba contro la volontà degli aventi diritto. Ad Aphrodisias, in Caria, alcuni documenti di II/III sec. d.C. (I.Aph2007 12.320, 322, 524, 526, 909) fanno ricorso al verbo $\dot{\alpha} \varphi \eta \rho \omega \hat{i} \zeta \omega$ per affermare che il monumento funebre sarà considerato "sacro agli eroi" al momento della sua chiusura, cioè una volta avvenuta la sepoltura delle persone menzionate nel documento come aventi diritto all'uso della tomba ${ }^{49}$. Tale strategia, che mira a rafforzare tramite la consacrazione della tomba il dispositivo giuridico a tutela della sua inviolabilità/inalienabilità, sembra emergere anche da un frammentario regolamento sepolcrale da Akmonia, in Frigia (MAMA VI 312). In Mesia inferiore, invece, nella regione di Philippopolis, il verbo $\dot{\alpha} \varphi \eta \rho \omega \hat{\zeta} \zeta \omega$ è impiegato in formule testamentarie molto più succinte, nelle quali l'oggetto dell'atto di eroizzazione non è la tomba, ma la persona che vi è sepolta. In due dei tre casi registrati, l'effetto del verbo $\dot{\alpha} \varphi \eta \rho \omega \hat{i} \zeta \omega$ ricade sulla stessa persona che compie l'azione, e che quindi si "autoeroizza" ${ }^{50}$. Ugualmente brevi sono le formule attestate sporadicamente a Chios, Perinthos, Trezene, Atene, e nella maggior parte dei documenti provenienti da Thera (I-III d.C.): in questi casi ci troviamo di fronte all'iniziativa di uno o più privati che sanciscono lo statuto eroico di un parente o di un servo defunti. Più elaborati sono invece i testi pubblici di Thera e Anaphe, nei quali la boule, da sola o con il dèmos, prende l'iniziativa di eroizzare una persona benemerita. Spesso questi documenti fanno riferimento ai meriti che giustificano tale iniziativa, accompagnano il verbo $\alpha \dot{\varphi} \varphi \rho \omega \hat{\zeta} \zeta \omega$

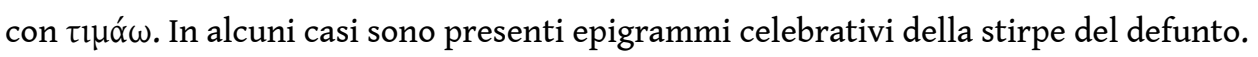

Il problema sollevato dalla documentazione imperiale concerne il differente valore dei lunghi regolamenti funerari di Aphrodisias (a cui andrà forse aggiunto il frammento da Akmonia) rispetto alle altre brevi iscrizioni che si limitano a commemorare l'eroizzazione del defunto. La prima tipologia fa esplicito riferimento al fatto che il documento iscritto sul monumento funebre costituisce l'originale di una risoluzione legale, la cui copia è stata depositata dal proprietario del sepolcro presso un archivio pubblico ${ }^{51}$. Il meccanismo religioso di tutela della tomba attivato dalla consacrazione del sepolcro a defunti eroizzati non fa che rafforzare i dispositivi legali attestati in numerosi testi funebri di età imperiale. Diverso sembra essere il caso delle epigrafi brevi. Nei documenti pubblici di Thera e Anaphe, l'atto di eroizzazione rimanda a una decisione pubblica effettuata dal Consiglio, talora insieme all'assemblea popolare, anche se il testo iscritto sul monumento funebre non riporta per esteso il decreto, limitandosi piuttosto a evocarne le conseguenze onorifiche. Tali organi pubblici potrebbero essere considerati come i garanti del rispetto degli onori eroici, ma il contenuto di tali onori ci sfugge completamente: come in altri casi di età imperiale, manca qualunque riferimento alla pratica di un culto eroico per i defunti, né è certo che l'eroizzazione pubblica implichi di per sé un ruolo attivo dello Stato nella costruzione o nella tutela di un monumento funebre per le persone onorate. Ancora più incerte sono le implicazioni dell'eroizzazione nella documentazione privata: se si

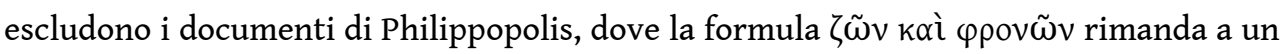
contesto testamentario ${ }^{52}$, le altre fonti non presentano alcun riferimento a un quadro giuridico nel quale l'eroizzazione potrebbe costituirsi come meccanismo di tutela del monumento funebre. In tali casi, si potrebbe dire che il verbo $\alpha \dot{\varphi} \varphi \rho \omega \hat{i} \zeta \omega$, non 
diversamente dal nome ท̆ $\rho \omega \varsigma$, assolva la propria funzione nell'onorare un defunto su un piano simbolico, evocando la potenza e l'inviolabilità di un eroe a un livello discorsivo, mentre le implicazioni giuridiche e/o rituali dello statuto eroico ci sfuggono interamente.

\section{Feste Hērōia}

Due testi epigrafici attestano l'uso della parola Hērōia per indicare feste per eroi, sia pure con valenze diverse. In un decreto onorifico attico del 137/6 a.C. ${ }^{53}$, l'onorando, un sacerdote di Asclepio, è elogiato per aver compiuto splendidi sacrifici in occasione delle feste Epidauria, Asklēpieia e Hērōia. In questo caso, il referente del culto sembra essere lo stesso Asclepio nella sua dimensione di eroe guaritore. Un documento di Cizico della fine del I sec. a.C., in onore di un membro dell'élite civica di nome Demetrios, definisce invece Hērōia una festa di ringraziamento istituita per il nonno dell'onorando, Asklepiades. Già membro dell'esercito che aveva difeso Cesare ad Alessandria nel 47 a.C., Asklepiades aveva ottenuto a suo tempo dalla propria città il titolo altamento onorifico di "fondatore", l'istituzione di una festa celebrata dai frequentatori del ginnasio e la costruzione di un hērōon, divenuto in seguito monumento funebre di tutta la famiglia ${ }^{54}$.

\section{Associazioni di Hērōistai}

Con il nome Hērōistai si autodefiniscono varie associazioni familiari i cui membri si organizzano in un koinon per commemorare alcuni membri defunti della famiglia. Un documento proveniente da una ignota località della valle del Kaystros, (Lidia sudoccidentale; SEG LVII 1188) ci offre una descrizione dettagliata dei meccanismi di attribuzione di onori eroici in una associazione organizzata attorno a un gruppo familiare allargato del II sec. a.C. ${ }^{55}$. Il decreto di un koinon tardo-ellenistico di Hērōistai ad Atene (57/6 a.C.) regola il pagamento delle quote di sottoscrizione per i membri dell'associazione. La natura familiare dell'associazione è rivelata dal fatto che il ruolo prestigioso di archieranistēs è probabilmente ricoperto dal figlio di Diotimos, una delle tre persone onorate dagli Hēröistai ${ }^{56}$. Da Mylasa proviene un testo frammentario non datato, menzionante un'associazione di Hēröistai e contenente il regolamento per un sacrificio e il riferimento a una statua ${ }^{57}$. Una base rinvenuta a Rodi tramanda una dedica al koinon dei Sōtēriastai ed Hērōistai da parte di un euergetēes di nome Zenodotos di Perge ${ }^{58}$, mentre dalla Beozia di età romana proviene una fondazione testamentaria contenente il regolamento interno di una synodos di Hērōistai, esposto dalla madre dei due giovani onorati, Epaminondas e Theokrines ${ }^{59}$. Un'associazione di Hērōistai sembra attestata anche nel villaggio di Apateria (valle del Kaystros, territorio di Efeso) ${ }^{60}$. Qui, un documento frammentario databile alla fine del I sec. d.c. ci informa sulla fondazione funeraria di un certo Peplos, proprietario di un hērōon per il quale predispone un dispositivo di tutela legale del monumento (linee 1-7), elenca le componenti del ricco arredo funerario (linee 7-17) e regola il meccanismo di accesso all'associazione responsabile per la cura della tomba e del culto (linee 17-20). Malgrado il suo stato lacunoso, dal testo si evince che l'associazione era costituita da philoi di Peplos, i cui nomi erano citati nella parte mancante dell'atto di fondazione; ciascun hērōo(i)stēs 
doveva essere sostituito alla sua morte dal figlio, o, se privo di prole, da un'altra persona, forse un parente ${ }^{61}$.

Il confronto con i meccanismi amministrativi e con le pratiche rituali di altre associazioni familiari dedite al culto dei defunti mostra una sostanziale somiglianza fra gli Hērōistai ed altre associazioni che non presentano un riferimento esplicito al culto eroico nel loro nome ${ }^{62}$. Come nel caso delle denominazioni della tomba, dunque, appare evidente che il ricorso alla sfera semantica eroica non costituisce di per sé un discrimine né sul piano cultuale, né su quello dell'organizzazione amministrativa dell'associazione.

\section{La formula ñ}

Lo spoglio della documentazione epigrafica ha permesso di individuare un numero esiguo di attestazioni dell'aggettivo ทं $\omega$ wïkóc. Solo in tre casi, l'aggettivo appare in

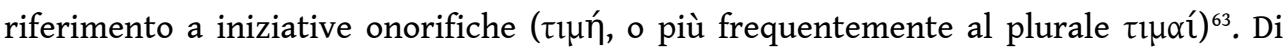
questi, la più antica attestazione risale al I sec. a.C. e proviene da una regione di confine del mondo greco: il regno di Commagene, dove i regolamenti rituali emessi dal re Antioco I parlano di $\dot{\eta} \rho \omega \ddot{k} \hat{\eta} \tau \imath \mu \eta^{64}$ e di $\varphi v ́ \sigma ı \zeta ~ \dot{\eta} \rho \omega \ddot{k} \eta^{65}$ per descrivere il proprio progetto di culto dinastico dei predecessori in una esplicita prospettiva di culto eroico di tipo greco $0^{66}$. Le due occorrenze di età imperiale si riferiscono agli onori funebri per il magistrato benefattore Memmios Nikandros a Delfi (FD III 1, 466; c. 125-150 d.C.) ${ }^{67}$ e per Euboulos figlio di Apollodoros, a Cnido, un atleta morto in giovane età e destinatario di

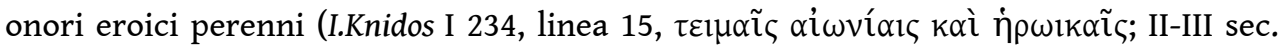
d.c.).

Le occorrenze letterarie della formula ทं $\rho \omega \ddot{k} \alpha \grave{~ \tau ı \mu \alpha i ́ ~ s o n o ~ p i u ̀ ~ n u m e r o s e, ~ m a ~ r e s t a n o ~ i n ~}$ ogni caso limitate e la loro cronologia appare particolarmente degna di interesse. A

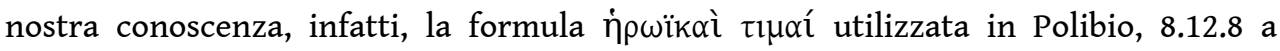

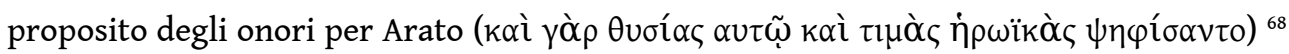
costituisce la sola attestazione precedente l'opera di Diodoro. Lo storico siceliota è in

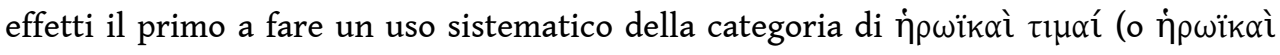
$\left.\theta v \sigma i \alpha_{1}\right)$, impiegandola con una certa frequenza per fare riferimento a onori cultuali accordati a personaggi mitici o a figure storiche del passato ${ }^{69}$. Per varie occorrenze è possibile ipotizzare che l'intento classificatorio di Diodoro abbia indotto l'autore ad applicare tale categoria di propria iniziativa, al fine di disambiguare casi in cui lo statuto cultuale della figura onorata era in effetti piuttosto fluido, o in sezioni della Biblioteca nelle quali il nostro autore presta una particolare attenzione, come nel caso dei tiranni sicelioti, alla costruzione di un modello carismatico di potere ${ }^{70}$. Lo stesso apporto personale di Diodoro emerge nel resoconto di culti accordati da popolazioni non greche, per le quali il culto eroico non poteva certo essere una soluzione percorribile per le comunità onoranti ${ }^{11}$. La sistematizzazione, riscontrabile in Diodoro,

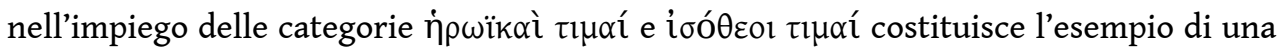
tendenza rintracciabile attraverso vari aspetti della cultura tardo-ellenistica: si tratta di un crescente interesse per le implicazioni teoriche degli onori rituali per benefattori umani, principalmente riscontrabile nei testi letterari, ma non priva di influssi sulla documentazione epigrafica. Tale interesse si traduce, nella Biblioteca di Diodoro come in 
altre opere storiografiche coeve, in un tentativo di classificazione della natura religiosa degli onori che non ha riscontro nella precedente documentazione epigrafica.

\section{Onori eroici}

L'analisi sin qui condotta ha offerto materiale utile a una riflessione sinottica. Nel tentativo di fornire una casistica che permetta di studiare in modo sistematico le varie manifestazioni di onori cultuali di tipo eroico e le loro premesse socio-politiche (in termini di destinatari, attori e contesti), procederemo alla definizione di quattro grandi categorie:

1) gli onori eroici per capi politici e benefattori locali, decretati e amministrati a livello istituzionale;

2) gli onori eroici accordati dalla polis a membri di famiglie dell'élite, i cui onori sono riflesso della loro distinzione sociale piuttosto che conseguenza diretta di azioni evergetiche compiute dagli onorandi;

3) gli onori eroici accordati a membri delle élites all'interno di associazioni private di pari;

4) gli onori per defunti privati per i quali l'iscrizione funeraria fornisce la definizione di

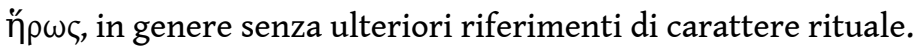

Tra i destinatari di onori eroici per capi politici possiamo distinguere tre categorie a seconda della posizione dell'onorando in relazione al mondo della polis: i sovrani (livello supra-polis); i grandi generali a capo di Leghe civiche regionali, appartenenti a una polis ma al contempo posti al di sopra di essa in virtù del loro statuto di comandanti federali; i grandi benefattori civici, la cui attività si iscrive nell'orizzonte di una città, sia pure con ricadute significative sulla sua politica estera.

L'intensa attività ecistica svolta dagli ultimi re argeadi e dai nuovi monarchi ellenistici aprì le porte all'istituzione di culti postumi ispirati alla tradizione coloniale dell'eroizzazione dei fondatori di nuove città, talora accompagnati dall'erezione di santuari collocati in posizione prominente all'interno della città ${ }^{72}$. Tuttavia, prima di Diodoro le fonti antiche non chiamano mai esplicitamente in causa la sfera semantica dell'ň $\rho \omega \varsigma$ per i sovrani defunti. Esse si limitano piuttosto a derivare dal rapporto religioso dio-eroe uno strumento per tradurre in termini di pratica onorifica la gerarchia politica fra i sovrani e i loro philoi e collaboratori ${ }^{73}$.

31 Un esempio paradigmatico del conferimento di onori eroici al comandante di una Lega interregionale è il già citato caso di Arato a Sicione nel 213/2 a.C. Gli onori postumi di Arato si inseriscono in una tradizione di grandi benefattori civici riconosciuti e onorati come nuovi fondatori di una città ${ }^{74}$. Allo stesso tempo, il loro contenuto non si differenzia nella sostanza dalle contemporanee iniziative messe in atto dalle città per $i$ sovrani evergeti ${ }^{75}$. Alla stessa categoria appartiene anche il dossier concernente un altro comandante della Lega Achea, Philopoimen, il cui corpo fu trionfalmente ricondotto a Megalopoli dopo la morte nel 183/2 a.C. e sepolto in un monumento eretto per l'occasione nell'agorà della madrepatria. Philopoimen ricevette anche una serie di onori rituali, elencati da una iscrizione di Megalopoli e confermati dalle fonti letterarie ${ }^{76}$. Di particolare interesse è il fatto che tali onori, che pure comprendevano una componente tipicamente eroica come quella della sepoltura all'interno delle mura cittadine, siano definiti concordemente dalle fonti come "uguali a quelli degli dei" ${ }^{7}$, 
dettaglio che attira l'attenzione sulla fluidità che caratterizza il rapporto fra onori "eroici" e "divini" nella documentazione di età ellenistica.

32 A un livello più basso di influenza politica si colloca l'operato di un benefattore locale eroizzato come Artemidoro di Perge a Thera ${ }^{78}$. Figura particolarmente attiva nella vita religiosa di Thera nella seconda metà del III sec. a.C. ${ }^{79}$, Artemidoro fondò un santuario rupestre dedicato a più divinità e localizzato all'ingresso settentrionale della città. Se l'iniziativa di Artemidoro è stata spesso intesa, in modo parziale, come la manifestazione di una devozione individuale, il nesso fra la sua attività e il quadro più ampio della vita di Thera - nella sua dimensione sia strettamente cultuale sia politica, come emerge dalle iniziative che coinvolgono gli onori per i Tolemei ${ }^{80}$ - è confermato dal fatto che per Artemidoro, la città di Thera istituì, con il consenso dell'oracolo di Delfi, un culto che lo definisce $\dot{\alpha} \theta \alpha$ óvatov $[\theta \varepsilon]$ ĩov n̆ $\rho \omega v^{81}$. Si tratta in questo caso dell'unico processo di eroizzazione noto sull'isola prima dell'età imperiale.

\section{Tendenze oligarchiche e la dimensione familiare dell'evergetismo pubblico}

33 A partire dal II sec. a.C., le istituzioni politiche della Grecia continentale e dell'Asia Minore conoscono un'evoluzione oligarchica, che a livello sociale si segnala nell'affermazione di una classe dirigente stabile, composta di poche famiglie capaci di guidare e controllare la vita politica e culturale della città ${ }^{82}$. In questo contesto si comprende perché, nei decreti onorifici dell'epoca, l'enumerazione dei meriti dell'onorando sia spesso accompagnata dalla celebrazione delle benemerenze passate della sua famiglia ${ }^{83}$. Specularmente, i meriti di una figura di spicco della comunità locale possono giustificare l'attribuzione di onori eroici a suoi familiari defunti. Questo processo si segnala particolarmente nel caso di figure la cui possibilità di partecipare attivamente alla vita sociale della città è limitata da fattori di genere (donne) $)^{84}$ e d'età (giovani morti prima, o all'inizio dell'età adulta).

All'interno di questa categoria si segnala una varietà di sfumature per quel che riguarda l'equilibrio fra l'iniziativa onorifica presa dalla famiglia dell'onorando e quella delle istituzioni civiche. Dove la documentazione è sufficientemente chiara, possiamo distinguere due casi. Al primo corrispondono onori stabiliti a spese della città. Oltre al testo di Itanos discusso in precedenza, possiamo citare IG XII 7, 447, da Aigiale di Amorgo, II-I s. a.C.: un epigramma sepolcrale che ricorda gli onori accordati dalla città al giovane Leonteus figlio di Eurydikos, morto dopo la fine dell'efebia, eroizzato pubblicamente e onorato con una tomba all'interno del ginnasio ${ }^{85}$. Al secondo sottogruppo appartengono gli onori istituiti da membri della famiglia dell'onorando e quindi approvati a livello pubblico. In questo caso, i fondi necessari per il culto sono privati, ma amministrati pubblicamente. Un caso significativo è offerto dalla già citata fondazione cultuale istituita, ancora ad Aigiale di Amorgo durante il II secolo, da Kritolaos, membro dell'élite civica e padre di Aleximachos, giovane morto durante l'efebia (IG XII 7 515). La sostenibilità di tale fondazione è garantita da un capitale di partenza donato da Kritolaos e mantenuto attraverso un sistema di prestiti a interesse rigorosamente definito nel documento e sottoposto a supervisione pubblica. 


\section{Fondazioni e associazioni private}

a.C. (IG II $^{2}$ 1326, linee 4-21), la lunga lista dei meriti del defunto Dionysios, già sacerdote tesoriere e benefattore dell'associazione, giustifica la sua eroizzazione - ancora una volta espressa nei termini di una procedura deliberativa attuata dall'associazione (linee 46-48) - e l'erezione del suo ritratto accando alla statua del dio. La centralità della famiglia di Dionysios nella gestione dell'associazione cultuale di Dioniso emerge chiaramente da questo decreto e da uno precedente, già emesso per lo stesso Dionysios in vita (IG II ${ }^{2} 1323 ; 185 / 4$ a.C. $)^{88}$, confermando perciò le somiglianze fra le dinamiche di eroizzazione all'interno dell'associazione dei Dionysiastai del Pireo e altre fondazioni familiari dell'epoca: Dionysios è stato responsabile della costruzione del santuario all'interno della proprietà familiare; una statua del padre si trova già accanto a quella di Dioniso al momento dell'erezione del nuovo ritratto del benefattore; i figli di Dionysios sono anch'essi membri del thiasos e uno di loro, Agathokles, è nominato sacerdote a vita al posto del padre. Il nesso fra l'evergetismo religioso individuale e l'istituzione di onori eroici si segnala come un ulteriore tratto comune alle più antiche fondazioni familiari, fra la fine del IV e il II s. a.C. In questi casi - come è ben illustrato dal famoso testamento di Epitteta a Thera - l'istituzione di onori eroici per un defunto segue l'atto di fondazione, da parte dell'onorando, di un culto familiare per una o più divinità. Nel tempo, a tale culto si affiancano quindi gli onori funebri per uno o più membri della famiglia ${ }^{89}$.

Risulta difficile spiegare in modo chiaro e univoco perché solo alcune associazioni sentirono il bisogno di esplicitare le motivazioni del conferimento di onori eroici ai loro membri defunti. Una spiegazione plausibile è che tale giustificazione si rendesse 
necessaria solo nel caso di associazioni composte da gruppi familiari allargati, la cui struttura complessa poteva richiedere una gestione più formale del processo di conferimento degli onori e dell'allocazione dei fondi comuni a tale scopo. In un gruppo familiare ristretto, tale formalismo avrebbe potuto essere semplicemente superfluo. D'altro canto, non possiamo escludere un'evoluzione diacronica: la moltiplicazione dei casi di eroizzazione privata dall'inizio dell'età ellenistica all'alta età imperiale sembra implicare una progressiva routinizzazione del processo di allargamento degli onori cultuali a nuovi membri della famiglia.

\section{La scissione fra immaginario dell'aldilà e rituale: ňpws nelle iscrizioni sepolcrali private}

Queste osservazioni ci permettono di passare all'ultima categoria che abbiamo definito all'inizio di questo tentativo di analisi sociologica: quella dei defunti i cui onori sembrerebbero distinguersi dalle più comuni pratiche funerarie solamente per la presenza della marca linguistica di n̆ $\rho \omega \varsigma$ nell'epigrafe sepolcrale. La grande maggioranza delle epigrafi di età ellenistica e imperiale si limita in effetti a menzionare il nome del defunto, spesso accompagnato da indicazioni sulla sua professione, gli anni vissuti, le cause della morte, il lutto dei familiari. La stele funebre è talora decorata con un'iconografia ispirata a una serie di tematiche ricorrenti, fra le quali dominano le scene di commiato, di banchetto e quelle raffiguranti il defunto con alcuni attributi che ne caratterizzano l'età, il genere o la professione. Su un numero cospicuo, ma comunque minoritario di stele, il nome del morto è accompagnato dall'apposizione

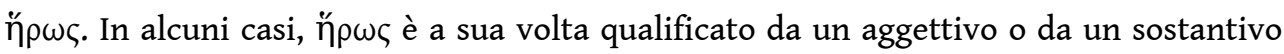

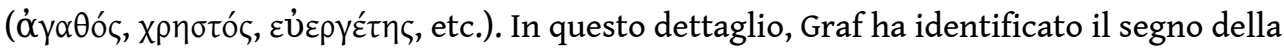
volontà dei viventi di affermare la convinzione che il defunto, divenuto n̆ $\rho \omega \varsigma$, non abbia perso la propria capacità di interagire in modo benevolo con la comunità dei suoi cari $^{90}$. Tale conclusione richiede tuttavia di essere sfumata alla luce della ricerca lessicografica da noi condotta, la quale ha rivelato che la presenza più o meno frequente di questi aggettivi dipende in primo luogo dall'usus epigrafico regionale.

In alcuni testi più elaborati, i riferimenti al morto e alla sua famiglia sono arrichiti dall'enunciazione di aspettative positive per il destino del defunto, per il quale si auspica o si prospetta un soggiorno felice nelle isole dei beati, fra gli dei o gli eroi, sull'Olimpo o in cielo. Wypustek ha ben rilevato la capacità di questi testi di assorbire $\mathrm{e}$ adattare a un registro popolare una lunga tradizione poetica e un immaginario dell'aldilà in precedenza limitato a strati elevati della società. L'autore sottolinea altresì che il carattere stereotipato dei motivi relativi al destino dei defunti non implica di per sé una perdita della loro pregnanza culturale: nella tarda età ellenistica e sotto l'Impero, un numero crescente di famiglie desidera appropriarsi di aspettative ultraterrene positive per i propri defunti, mettendo in luce una pluralità di possibili immaginari dell'aldilà che, almeno fino all'avvento del Cristianesimo, coesistono con immagini più tradizionali dell'Ade, senza implicare un effettivo cambiamento di paradigma ${ }^{91}$.

41 Alcuni indizi inducono dunque a condividere la posizione più recente della critica, intesa a superare il verdetto di una semplice banalizzazione della categoria eroica a favore di un modello intepretativo capace di includere fra le espressioni religiose di questa categoria anche le sue manifestazioni più semplici e popolari. D'altro canto, 
altre considerazioni ci invitano a sfumare le implicazioni di tale cambio di paradigma. In primo luogo, la povertà e la ripetitività dei supporti sepolcrali nei quali il defunto è definito ท̆ $\rho \omega \varsigma$, e talora si vede attribuito un destino ultraterreno simile a quello degli eroi mitici, non permette di supporre che la persona onorata abbia ricevuto un trattamento rituale differente da quello di qualunque altro defunto. Inoltre, quando una stele sepolcrale per un defunto definito ň $\rho \omega \varsigma$ presenta decorazioni iconografiche, queste in linea di massima non si differenziano dalla gamma di soggetti impiegati per stele non caratterizzate da una marca linguistica eroica. Per finire, come si è visto, molte occorrenze dei termini $\dot{\eta} \rho \tilde{\omega} o v$ e $\dot{\alpha} \varphi \eta \rho \omega \hat{i} \zeta \omega$ nella documentazione di età imperiale rispondono allo scrupolo di assicurare una tutela di natura religiosa al monumento funebre piuttosto di esprimere l'eccezionalità del trattamento rituale di un defunto.

Pur riconoscendo l'intrinseca valenza religiosa della parola ท̆ $\rho \omega \varsigma$ anche nelle sue manifestazioni meno monumentali, è dunque necessario ricondurre la nostra documentazione nell'alveo del panorama sociologico e diacronico che abbiamo definito in precedenza. Da un lato, in età ellenistica, la pratica funeraria privata può attingere a meccanismi organizzativi e a un vocabolario propri della sfera pubblica; inoltre, forme di immaginario tradizionalmente legate a classi sociali e a registri discorsivi alti possono essere selezionate e adattate ai contesti di fruizione e alle aspettative quotidiane di un numero crescente di famiglie appartenenti alla massa anonima della popolazione. D'altro canto, questa moltiplicazione e diffusione dei contesti di applicazione della sfera semantica di ท̆ $\rho \omega \varsigma$, che potremmo definire, pur in modo sommario, come una estenzione dall'alto verso il basso della società, si accompagna a un decisivo svuotamento del contenuto rituale eccezionale tradizionalmente associato agli onori eroici.

Riprendendo le osservazioni sociologiche che abbiamo fatto a proposito del passaggio del processo eroizzante da occasionale (ed eccezionale) a sistematico all'interno delle associazioni costituite da famiglie delle élites locali, possiamo cercare di restituire anche alle iniziative apparentemente più banali un po' della loro profondità sociale e culturale. In questi termini, potremmo affermare che il privato commemorato come ท̆ $\rho \omega \varsigma$ su una lapide o un altare funebre è in primo luogo un defunto, ma uno al quale $\mathrm{i}$ cari rimasti in vita desiderano attribuire uno statuto onorifico capace di evocare, in maniera implicita o esplicita, non solo l'inviolabilità della sepoltura, ma anche una serie di aspettative ultraterrene e di capacità di interazione con i vivi fuori della norma.

\section{Considerazioni conclusive}

La nostra indagine lessicografica ha rivelato differenti livelli di concentrazione della parola n̆ $\rho \omega \varsigma$ e dei suoi derivati in specifici contesti cronologici, geografici e mediali. In questo senso, la categoria di usus deve essere tenuta in considerazione per evitare semplificazioni e generalizzazioni. Nella maggior parte dei casi, le fonti non ci aiutano a individuare le ragioni contingenti per cui una persona o un gruppo decisero di fare appello esplicito alla categoria eroica per accrescere il prestigio e l'onore riconosciuti a un benefattore, a un membro del gruppo, o a se stessi. Tuttavia, due aspetti fondamentali possono essere evidenziati.

In primo luogo, la sfera semantica di n̆ $\rho \omega \varsigma$, nei suoi impieghi in contesto rituale, giuridico e legato alla rappresentazione delle aspettative per l'aldilà, rimanda in modo 
sostanziale a una sfera di applicazione funebre ${ }^{92}$. Questa affermazione resta valida anche qualora l'istituzione di onori eroici sia decisa prima della morte dell'onorando, come certamente si verifica nelle fondazioni testamentarie in cui un privato instituisce e regola gli onori funebri futuri per se stesso e i propri familiari. La nostra seconda riflessione conclusiva concerne la questione del posizionamento della sfera semantica di ň $\rho \omega \varsigma$ nel più ampio quadro della religione greca nel mondo ellenistico-imperiale. Gli studi degli ultimi due decenni hanno ribaltato la visione tradizionale che vedeva nell'età ellenistica un periodo di decadenza del culto eroico. Al contrario, in età ellenistica e imperiale la categoria eroica sembra avere conosciuto un significativo e ininterrotto successo, riscontrabile sia al livello degli eroi mitologici o "funzionali"

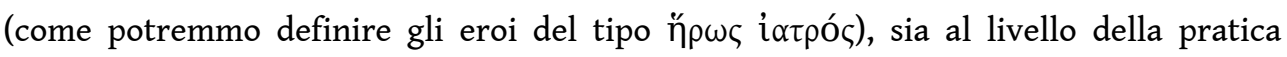
onorifica funeraria.

Se consideriamo tale fenomeno dal punto di vista degli strati alti della società, concorderemo con la critica recente nell'attribuire alle élites locali una posizione importante nella promozione sia dei culti associati a eroi mitici, sia degli onori eroici per i defunti. Non diversamente che per l'età arcaica, la valorizzazione del culto degli eroi mitici sembra avere, ancora in età ellenistica e imperiale, un ruolo centrale nella vita e nelle strategie di auto-rappresentazione di un gruppo sociale all'interno del suo ambiente d'azione: si pensi alle famiglie dell'élite che rivendicano una parentela con gli eroi del mito, o alle istituzioni civiche che negli eroi dell'epopea trovano gli strumenti per cercare tutela nei momenti di crisi, rafforzare la coesione interna della comunità e accrescere il proprio prestigio internazionale. Quanto agli onori eroici per i defunti, essi offrono alle famiglie delle élites gli strumenti per cementare i propri vincoli interni e per competere all'esterno con altri gruppi dominanti.

Se ora ci muoviamo verso gli strati bassi della popolazione, la discussione della valenza culturale e religiosa di n̆ $\rho \omega \varsigma$ ci espone a una serie di problematiche più complesse, perché più evanescenti sono le fonti a nostra disposizione. Durante l'età ellenistica $\mathrm{e}$ soprattutto durante l'alta età imperiale, all'interno dell'ampia gamma di onori

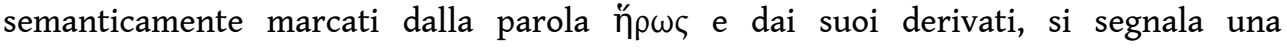
significativa distinzione fra onori monumentalmente e ritualmente elaborati da una parte e, dall'altra, un numero crescente di casi che non sembrano affatto differenziarsi, sul piano monumentale o rituale, dalla cura riconosciuta a semplici defunti. D'altro canto, la valenza religiosa della sfera semantica di n̆ $\omega \varsigma$ non può essere esclusa tout court a causa dell'assenza di processi di monumentalizzazione e di particolare ritualizzazione degli onori funebri. I ceti meno abbienti non avevano né le risorse economiche, né lo stimolo alla competizione sociale che giustificano la monumentalizzazione degli onori funebri presso le élites. Presso questi attori, la vitalità della categoria eroica sembra manifestarsi piuttosto al livello giuridico della tutela delle sepolture, nonché sul piano dell'immaginario dell'aldilà, come mostrano le aspettative che un numero crescente di famiglie esprime a proposito di un destino ultraterreno felice per i propri cari defunti.

La valenza onorifica della categoria di й $\rho \omega \varsigma$ non dovrebbe dunque essere considerata, a nessun livello sociale, come alternativa, e nemmeno come separata da una valenza religiosa, sebbene tale significato religioso possa spesso esprimersi in un senso lato e non strettamente associato alla sfera della pratica rituale. Nell'interpretare il fenomeno degli onori eroici nel mondo greco ellenistico, sarà utile ricordare, in conclusione, i rischi di operare una tale separazione per una civiltà che nella sua lingua non ha 


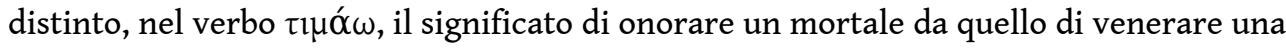
divinità.

ALcock 1991: S.E. Alcock, «Tomb Cult and the Post-Classical Polis», AJA 95 (1991), 447-467.

Alcock 1997: S.E. Alcock, «The Heroic Past in a Hellenistic Present», in P. Cartledge et al. (eds), Hellenistic Constructs: Essays in Culture, History, and Historiography, Berkeley - Los Angeles - London 1997, 20-34.

HARTER-UibOPUU 2010: K. Harter-Uibopuu, «Erwerb unr Veräusserung von Grabstätten im griechisch-römischen Kleinasien am Beispiel der Grabinschriften aus Smyrna», in G. Thür (hrsg.), Symposion 2009, Wien 2010, 247-270.

Harter-Uibopuu 2011: K. Harter-Uibopuu, «Money for the Polis: Public Administration of Private Donations in Hellenistic Greece», in O.M. van Nijf, R. Alston (eds), Political Culture in the Greek City After the Classical Age, Leuven - Paris - Walpole, MA 2011, 119-139.

KUBIŃSKA 1968: J. Kubińska, Les monuments funéraires dans les inscriptions grecques de l'Asie Mineure, Varsovie 1968.

Kurtz - Boardman 1971: D.C. Kurtz, J. Boardman, Greek Funerary Practice, London - New York 1971. ROBERT 1930: L. Robert, «Études d'épigraphies grecques», RPh 4 (1930), 25-60 [= OMS II, 1125-1160]. Robert 1966: L. Robert, Documents de l'Asie Mineure méridionale. Inscriptions, monnaies et géographie, Genève - Paris 1966.

STRUBBE 1997: J.H.M. Strubbe, APAI EПITYMBIOI. Imprecations Against Desecrators of the Grave in the Greek Epitaphs of Asia Minor: A Catalogue, Bonn 1997.

Strubbe 2004: J.H.M. Strubbe, «Cultic Honours for Benefactors in the Cities of Asia Minor», in L. de Ligt, E.A. Hemelrijk, H.W. Singor (eds), Roman Rule and Civic Life: Local and Regional Perspectives, Amsterdam 2004, 315-330.

\section{BIBLIOGRAFIA}

AGRW: Associations in the Greco-Roman World: An Expanding Collection of Inscriptions, Papyri, and Other Sources in Translation, (http://philipharland.com/greco-roman-associations).

ARNAOUTOGLOU 1994: I. Arnaoutoglou, «APXEPANI $\Sigma T H \Sigma$ and Its Meaning in Inscriptions», ZPE 104 (1994), 107-110.

ARNAOUTOGLOU 2003: I. Arnaoutoglou, Thusias heneka kai sunousias. Private Religious Associations in Hellenistic Athens, Athens 2003.

BODDEZ 2016: Th. Boddez, «Entre le roi et la cité. Remarques sur le développement des cultes héroïques entre 336 et 150», Erga-Logoi 4.2 (2016), 75-116.

BOEHRINGER 1996: D. Boehringer, «Zur Heroisierung historischer Personlichkeiten bei den Griechen», in M. Flashar et al. (hrsg.), Retrospektive. Konzepte von Vergangenheit in der Griechischrömischen Antike, München 1996, 37-61. 
BOEHRINGER 2001: D. Boehringer, Heroenkulte in Griechenland von der geometrischen bis zum klassischen Zeit. Attika, Argolis, Messenien, Berlin 2001.

BOUSQUET 1966: Bousquet, «Inscriptions de Delphes», BCH 90.2 (1966), 428-446.

BURASELIS 2003: K. Buraselis, «Political Gods and Heroes or the Hierarchization of Political Divinity in the Hellenistic World», in A. Barzanò et al. (a cura di), Modelli eroici dall'Antichità alla cultura europea, Roma 2003, 185-197.

BURASELIS et al. 2004: K. Buraselis et al., «Heroisierung und Apotheose», ThesCRA II (2004), 125-214.

CAMPANELli 2012: S. Campanelli, «Eroizzazione e proprietà terriera nel "Testamento di Epikrates". Per una proposta di lettura delle fondazioni cultuali di carattere familiare», Hormos 4 (2012), 69-83.

CAMPANELli 2017: S. Campanelli, «Family Cult Foundations in the Hellenistic Age: Family and Sacred Space in a Private Religious Context», in M. Hilgert (ed.), Understanding Material Text Cultures: A Multidisciplinary View, Berlin 2017, 131-202.

CANEVA 2020a: S.G. Caneva, «Introduction: A Ritual and Material Turn in the Study of Cultic Honours for Hellenistic Political Leaders», in S.G. Caneva (ed.), The Materiality of Hellenistic Ruler Cults, Liège 2020, 9-18.

CANEVA 2020b: S.G. Caneva, «Le rôle du gymnase: espace, rituels et acteurs», in G. Lenzo, Ch. Nihan, M. Pellet (eds), Les cultes aux rois et aux héros dans l'Antiquité: continuités et changements à l'époque hellénistique (ORA), Tübingen 2020.

CANEVA 2021: S.G. Caneva, «Isotheoi ed heroikai timai in Diodoro», Hesperia 37 (2021).

CANEVA, in preparazione: S.G. Caneva, «Eroizzati in vita? Hagnon (Amphipolis), Adeimantos (Atene), Nikias (Cos) e Augusto (Alessandria) riconsiderati».

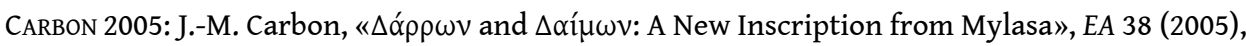
$1-6$.

CARBON - PirenNe-Delforge 2013: J.-M. Carbon, V. Pirenne-Delforge, «Priests and Cults in Three Hellenistic Families», in M. Horster, A. Klöckner (eds), Cities and Priests: Cult Personnel in Asia Minor and the Aegean Islands from the Hellenistic to the Imperial Period, Berlin 2013, 65-120.

CGRN: J.-M. Carbon, S. Peels, V. Pirenne-Delforge, A Collection of Greek Ritual Norms (CGRN), Liège 2016- (http://cgrn.ulg.ac.be).

CHANIOTIS 2004: A. Chaniotis, «New Inscriptions from Aphrodisia (1995-2001)», AJA 108 (2004), 377-416.

CHANIOTIS 2018: A. Chaniotis, Age of Conquests: The Greek World from Alexander to Hadrian, Cambridge, MA 2018.

CHIRICAT 2005: É. Chiricat, «Funérailles publiques et enterrement au gymnase à l'époque hellénistique», in P. Fröhlich, Ch. Müller (éds), Citoyenneté et participation à la basse époque hellénistique, Genève - Paris 2005, 121-144.

CHRUBASIK - KING 2017: B. Chrubasik, D. King (eds), Hellenism and the Local Communities of the Eastern Mediterranean. 400 BCE-250 CE, Oxford - London 2017.

Clay 2004: D. Clay, Archilochos Heros: The Cult of Poets in the Greek Polis, Washington 2004.

CURRIE 2005: B. Currie, Pindar and the Cult of Heroes, Oxford 2005. 
DE PolignaC 1995: F. de Polignac, Cults, Territory, and the Origins of the Greek City-State, Chicago London 1995.

DE VISSCHER 1963: F. De Visscher, Le droit des tombeaux romains, Milano 1963.

DiMITROVA 2002: N. Dimitrova, «Inscriptions and Iconography in the Monuments of the Thracian Rider», Hesperia 71.2 (2002), 209-229.

EITREM 1912: S. Eitrem, «Heros», RE VIII.1 (1912), coll. 1111-1145.

EKROTH 2002: G. Ekroth, The Sacrificial Rituals of Greek Hero-Cults in the Archaic to the Early Hellenistic Periods, Liège 2002.

EKROTH 2007: G. Ekroth, «Heroes and Hero-Cults», in D. Ogden (ed.), A Companion to Greek Religion, Oxford 2007, 100-114.

ЕкRотн 2009: G. Ekroth, «The Cult of Heroes», in S. Albersmeier (ed.), Heroes: Mortals and Myths in Ancient Greece, Baltimore 2009, 120-143.

EкRотн 2015: G. Ekroth, «Heroes - Living or Dead? », in E. Eidinow, J. Kindt (eds), The Oxford Handbook of Ancient Greek Religion, Oxford 2015, 383-396.

FABRICIUS 1999: J. Fabricius, Die hellenistischen Totenmahlreliefs. Grabrepräsentation und Wertvorstellungen in ostgriechischen Städten, München 1999.

FABRICIUS 2010: J. Fabricius, «Zwischen Konvention und Tabu - Zum Umgang mit Heroenehrungen in hellenistischen Poleis», in M. Meyer, R. von den Hoff (hrgg.), Helden wie sie. Übermensch - Vorbild - Kultfigur in der griechischen Antike, Freiburg 2010, 257-293.

FABRICIUS 2016: J. Fabricius, «Hellenistic Funerary Banquet Reliefs: Thoughts on Problems Old and New», in C.M. Draycott, M. Stamatopoulou (eds), Dining and Death: Interdisciplinary Perspectives on the 'Funerary Banquet' in Ancient Art, Burial and Belief, Leuven - Paris - Bristol 2016, 33-69.

FERRARY 2005: J.-L. Ferrary, «Les Grecs des cités et l'obtention de la ciuitas Romana», in P. Fröhlich, Ch. Müller (éds), Citoyenneté et participation à la basse époque hellénistique, Genève - Paris 2005, 51-75.

FORSTER 2018: F.R. Forster, Die Polis im Wandel. Ehrendekreten für eigene Bürger im Kontext der hellenistischen Polisgesellschaft, Berlin 2018.

FoUCART 1918: P. Foucart, Le culte des héros chez les Grecs, Paris 1918.

FRASER 1957: P.M. Fraser, Boeotian and West Greek Tomb-stones, Lund 1957.

FRASER 1977: P.M. Fraser, Rhodian Funerary Monuments, Oxford 1977.

FRöLICH 2013a: P. Frölich, «Funérailles publiques et tombeaux monumentaux intra-muros dans les cités grecques à l'époque hellénistique», in M.-Cl. Ferriès et al. (éds), Forgerons, élites et voyageurs d'Homère à nos jours. Hommages en mémoire d'Isabelle Ratinaud-Lachkar, Grenoble 2013, 227-309.

FRÖLICH 2013b: P. Frölich, «Un décret de Messène accordant des honneurs post mortem», in J.-Ch. Couvenhes (éd.), L'Hellénisme, d'une rive à l'autre de la Méditerranée. Mélanges offerts à André Laronde, Paris 2013, 241-266.

GAUTHIER 1985: Ph. Gauthier, Les cités grecques et leurs bienfaiteurs (IV ${ }^{e}-I^{\text {er }}$ siècle avant J.-C.). Contribution à l'histoire des institutions, Paris 1985.

GHERCHANOC 2012: Fl. Gherchanoc, L'oikos en fête. Célébrations familiales et sociabilité en Grèce ancienne, Paris 2012. 
GLEASON 2010: M. Gleason, «Making Space for Bicultural Identity: Herodes Atticus Commemorates Regilla» in T. Whitmarsh (ed.), Local Knowledge and Microidentities in the Imperial Greek World, Cambridge 2010, 125-162.

GORRINI 2013: M.E. Gorrini, Eroi salutari dell'Attica. Per un'archeologia dei cosiddetti culti eroici salutari della regione, Roma 2013.

GoUKOWSKI 1976: Diodore de Sicile, Bibliothèque historie. Livre XVII. Texte établi et traduit par P. Goukowski, Paris 1976.

GRAF 1985: F. Graf, Nordionische Kulte. Religionsgeschichtliche und epigraphische Untersuchungen zu den Kulten von Chios, Erythrai, Klazomenai und Phokaia, Genève 1985.

GRAF 1995 : F. Graf, «Bemerkungen zur bürgerlichen Religiosität im Zeitalter des Hellenismus», in M. Wörrle, P. Zanker (hrsg.), Stadtbild und Bürgerbild im Hellenismus, München 1995, 103-114.

GRAF 2005: F. Graf, «Hero Cult», in Brill's New Pauly 6 (2005), coll. 247-251.

HABICHT 1995: Ch. Habicht, «Ist eine 'Honoratiorenregime' das Kenzeichen des Stadt im späteren Hellenismus?», in M. Wörrle, P. Zanker (hrsg.), Stadtbild und Bürgerbild im Hellenismus, München 1995, 87-92.

HARTER-UIBOPUU 2014: K. Harter-Uibopuu, «Tote soll man ruhren lasse... Verbote und Strafen zur Sicherung von Gräbern am Beispiel des Inschriften von Ephesos», in J. Fischer (hrsg.), Der Beitrg Kleinasiens zur Kultur- und Geistesgeschichte der griechisch-römischen Antike, Wien 2014, 157-180.

HARTER-UIBOPUU - WIEDERGUT 2014: K. Harter-Uibopuu, K. Wiedergut, «Niemand anderer soll hier bestattet werden - Grabschutz im Kaiserlichen Milet», in G. Thür (hrsg.), Tagungen des Symposions “Grabritual und Jenseitsvorstellung”, Wien 2014, 147-172.

HeLmis 2003: A. Helmis, «Entre les vivants et les morts. La fondation à la mémoire d'Aleximachos fils de Critolaos (IG XII.7, 515 ; IIe siècle av. J.-C.)», in G. Thür, F.J. Fernández-Nieto (hrsg.), Symposion 1999, Köln 2003, 463-480.

HERMANN - MALAY 2007: P. Hermann, H. Malay, New Documents from Lydia, Wien 2007.

HERMANN - POLATKAN 1969: P. Herrmann, K.Z. Polatkan, Das Testament des Epikrates und andere neue Inschriften aus dem Museum von Manisa, Wien 1969.

HUGHES 1999: D.D. Hughes, «Hero Cult, Heroic Honors, Heroic Dead: Some Developments in the Hellenistic and Roman Periods», in R. Hägg (ed.), Ancient Greek Hero Cult, Stockholm 1999, 165-175. HUGHES 2019: D.D. Hughes, «The Cult of Aratus at Sicyon (Plutarch, Aratus, 53)», Kernos 32 (2019), 119-150.

JONES 2008: Ch.P. Jones, «A Hellenistic Cult-Association», Chiron 38 (2008), 195-204.

JoNES 2010: Ch.P. Jones, New Heroes in Antiquity: From Achilles to Antinoos, Cambridge, MA - London 2010.

KAMPS 1937: W. Kamps, «Les origines de la fondation cultuelle dans la Grèce ancienne», Archives d'Histoire du Droit Oriental 1 (1937), 145-179.

KeIL - von Premerstein 1914: J. Keil, A. von Premerstein, Bericht über eine dritte Reise in Lydien und den angrenzenden Gebieten Ioniens, ausgeführt $1911 \mathrm{im}$ Auftrage der Kaiserlichen Akademia der Wissenschaften, Wien 1914.

KIMMEL-CLAUZET 2013: Fl. Kimmel-Clauzet, Morts, tombeaux et cultes des poètes grecs, Bordeaux 2013. 
KLOPPENBORG - AscoUgh: J.S. Kloppenborg, R. Ascough, Greco-Roman Associations: Texts, Translations, and Commentary, I. Attica, Central Greece, Macedonia, Thrace, Berlin 2011.

LATTIMORE 1942: R. Lattimore, Themes in Greek and Latin epitaphs, Urbana, IL 1942.

LAUM 1914: B. Laum, Stiftungen in der griechischen und romischen Antike. Ein Beitrag zur Antiken Kulturgeschichte, Leipzig - Berlin, 1914.

LAZZARINI 1991: S. Lazzarini, Sepulchra familiaria. Un'indagine epigrafico-giuridica, Padova 1991.

LEBRIS 2001: A. Lebris, La mort et les conceptions de l'au-delà en Grèce ancienne à travers les épigrammes funéraires: étude d'épigrammes d'Asie mineure de l'époque hellénistique et romaine, Paris 2001.

LESCHHORN 1984: W. Leschhorn, Gründer der Stadt. Studien zu einem politisch-religiösen Phänomen der griechischen Geschichte, Wiesbaden 1984.

MALKIN 1987: I. Malkin, Religion and Colonization in Ancient Greece, Leiden - New York 1987.

MALKIN 1993: I. Malkin, «Land Ownership, Territorial Possession, Hero Cult, and Scholarly Theory», in R.M. Rosen, J. Farrel (eds), Nomodeiktes. Greek Studies in Honor of Martin Ostwald, Ann Arbor 1993, 225-234.

MANNZMANN 1962: A. Mannzmann, Griechische Stiftungsurkunden. Studie zu Inhalt und Rechtsform, Münster 1962.

MARI 2012: M. Mari, «La morte, il tempo, la memoria. Funerali pubblici e calendario civico nella Grecia antica», in V. Nizzo, L. La Rocca (a cura di), Antropologia e archeologia a confronto: rappresentazioni e pratiche del sacro, Roma 2012, 167-187.

MigeOTTE 2013: L. Migeotte, «Les souscriptions dans les associations privées», in P. Frölich, P. Hamon (éds), Groupes et associations dans les cités grecques (III' siècle av. J.-C. - II siècle apr. J.-C.), Genève 2013, 112-127.

MiккоLA 2008: M. Mikkola, «Heroa as Described in the Ancient Written Sources», in L. PietiläCastrén, V. Vahtikari (eds), Grapta Poikila II: Saints and Heroes, Athens 2008, 1-32.

MitTAG 2011: P.-F. Mittag, «Zur Entwicklung des „Herrscher-“- und „Dynastiekultes“ in Kommagene», in L.-M. Günther, S. Plischke (hrgg.), Studien zum vorhellenistischen und hellenistischen Herrscherkult: Verdichtung und Erweiterung von Traditionsgeflechten, Berlin 2011, 141-160.

Muccioli 2011: F. Muccioli, «Il culto del sovrano di epoca ellenistica e i suoi prodromi. Tre casi paradigmatici: Ierone I, Lisandro, la tirannide di Eraclea Pontica», in G. Cecconi, C. Gabrielli (a cura di), Politiche religiose nel mondo antico e tardoantico. Poteri e indirizzi, forme del controllo, idee e prassi di tolleranza, Bari 2011, 97-132.

MUCCIOLI 2014: F. Muccioli, «Cultes héroïques et cultes divins aux IV ${ }^{\mathrm{e}}$ et III ${ }^{\mathrm{e}}$ siècles av. J.-C. Tradition, innovation et reflets littéraires», Mythos 8 (2014), 13-34.

NocK 1944: A.D. Nock, «The cult of Heroes», HTR 37 (1944), 141-174 [= Essays on Religion and the Ancient World, Oxford 1972, II, 575-602].

NocK 1957: A.D. Nock, «Deification and Julian», JRS 47 (1957), 115-123 [= Essays on Religion and the Ancient World, Oxford 1972, II, 833-846].

PAlagia 1991: O. Palagia, "Cult and Allegory: The Life Story of Artemidoros of Perge», in J.M. Sanders (ed.), ФI $\Lambda \Lambda \mathrm{AK} \Omega$ N. Laconian Studies in Honour of Hector Catling, London 1992, 171-177.

PARROT 1939: A. Parrot, Maledictions et violations de tombes, Paris 1939. 
PERES 2003: I. Peres, Griechische Grabinschriften und neutestamentliche Eschatologie, Tübingen 2003.

PIRROTTA 2009: S. Pirrotta (ed.), Plato comicus: die fragmentarischen Komödien: ein Kommentar, Berlin 2009.

PfuHL - MoEBius 1977-1979: E. Pfuhl, H. Möbius, Die ostgriechischen Grabreliefs, Mainz 1977-1979.

RADULOVA 2016: L. Radulova, «Iura sepulcrorum nella Moesia Inferior: la realizzazione di un fenomeno romano in un ambito greco-trace», RIDA 63 (2016), 197-213.

RADULOVA - SASSU 2015: L. Radulova, R. Sassu, «Funerary Imprecations in the Balcan Provinces», AJIS 4.1 (2015), 469-478.

RAMSAY 1895: W.M. Ramsay, The Cities and Bishoprics of Phrygia, being an Essay of the Local History of Phrygia from the Earliest Times to the Turkish Conquest, Oxford 1895.

RITTI 2004: T. Ritti, «Iura sepulchrorum a Hierapolis di Frigia nel quadro dell'epigrafia sepolcrale microasiatica. Iscrizioni edite e inedite», in S. Panciera (a cura di), Libitina e dintorni. Libitina e i luci sepolcrali. Le Leges Libitinariae campane. Iura sepulchrorum: vecchie e nuove iscrizioni, Roma 2004, 455-634.

ROBERT 1969: L. Robert, «Les inscriptions», in J. Des Greniers et al. (éds), Laodicée du Lycos. Le Nymphée. Campagnes 1961-1963, Québec - Paris 1969, 247-389.

ROBERT 1978: L. Robert, «Malédictions funéraires grecques», Comptes rendus des séances de l'Académie des Inscriptions et Belles Lettres 122.2 (1978), 241-289.

ROHDE 1898²: E. Rohde, Psyche. Seelencult und Unsterblichkeitsglaube der Griechen, Freiburg $1898^{2}$.

SAMSARIS 1989: D.C. Samsaris, «La Vallée du Bas-Strymon à l'époque impériale. Contribution épigraphique à la topographie, l'onomastique, l'histoire et aux cultes de la province romaine de Macédoine», Dodona 18.1 (1989), 203-382.

SCHÖRNER 2007: H. Schörner, Sepulturae graecae intra urbem. Untersuchungen zum Phänomen der intraurbanen Bestattungen bei den Griechen, Münster - Möhnesee 2007.

SFAMENI-GASPARRO 1997: G. Sfameni-Gasparro, «Daimon and Tyche in the Hellenistic Religious Experience», in P. Bilde et al. (eds), Conventional Values of the Hellenistic Greeks, Aahrus 1997, 67-109.

SHERWIN-WHITE 1977: S. Sherwin-White, «Inscriptions from Cos», ZPE 24 (1977), 205-217.

SÈVE 1996: M. Sève, «Un enterrement public dans une épigramme d'Aigialè d'Amorgos (IG XII 7, 447)», RÉG 109 (1996), 683-688.

SNODGRASS 1982: A. Snodgrass, «Les origines du culte des héros dans la Grèce antique», in G. Gnoli, J.-P. Vernant (éds), La mort, les morts dans les sociétés anciennes, Cambridge - Paris 1982, 107-119.

Sosin 2014: S.J.D. Sosin, «Endowments and Taxation in the Hellenistic Period», AncSoc 44 (2014), 43-89.

STRUBBE 1991: J.H.M. Strubbe, «Cursed Be He That Moves My Bones», in C.A. Faraone, D. Obbink (eds), Magika Hiera. Ancient Greek Magic and Religion, New York - Oxford 1991, 33-59.

TulLy 2014: J. Tully, «Artemidoros' Temenos as a Memorial of Hellenistic Thera», in J. Bonnin, E. Le Quéré (éds), Pouvoir, îles et mer. Formes de l'hégémonie dans les Cyclades antiques (VII ${ }^{e}$ s. a.C. - III ${ }^{e}$ s. a.C.), Bordeaux - Paris 2014, 190-202.

VAN BREMEN 1996: R. van Bremen, The Limits of Participation: Women and Civic Life in the Greek East, Amsterdam 1996. 
VON MANGOLD 2013: B. von Mangold, Griechische Heroenkultstätten in klassischer und hellenistischer Zeit. Untersuchungen zu ihrer äußeren Gestaltung, Ausstattung und Funktion, Tübingen - Berlin 2013. VON WiLAmowitz-Moellendorff 1932: U. von Wilamowitz -Moellendorff, Die Glaube der Hellenen 2, Berlin 1932.

VOUTIRAS 2010: E. Voutiras, «Reiterheroen auf griechischen Weihreliefs des 5. und 4. Jahrhunderts vor Ch.», in M. Meyer, R. von den Hoff (hrgg.), Helden wie sie. Übermensch - Vorbild - Kultfigur in der griechischen Antike, Freiburg 2010, 85-106.

WAGNER - PETZL: J. Wagner, G. Petzl, «Relief- und Inschriftfragmente des kommagenischen Herrscherkultes aus Ancoz», in E. Winter (hrsg.), Neue Forschungen zur Religionsgeschichte Kleinasiens, Bonn 2003, 85-96.

WITTENBURG 1990: A. Wittenburg, Il testamento di Epikteta, Trieste 1990.

WYPUSTEK 2013: A. Wypustek, Images of Eternal Beauty in Funerary Verse Inscriptions of the Hellenistic and Greco-Roman Periods, Leiden - Boston 2013.

ZeLNICK-ABRAMOVITZ 2015: R. Zelnick-Abramovitz, «Whose Grave Is This? The Status of Grave Plots in Ancient Greece», Dike 18 (2015), 51-95.

\section{NOTE}

1. Il presente lavoro è frutto della collaborazione e della discussione fra i due autori: i parr. 1-6 sono stati redatti da A. Coppola; i parr- 7-13 da S. Caneva. Lo spoglio sistematico della documentazione epigrafica si è svolto combinando le pubblicazioni cartacee con i portali digitali del Packard Humanities Institute (PHI; https://inscriptions.packhum.org) e del progetto A Collection of Greek Ritual Norms (CGRN; http://cgrn.ulg.ac.be/) (consultazione aggiornata al giugno 2019).

2. Sui processi di eroizzazione pubblica in età ellenistica e imperiale, vd. B OEHRINGER 1996 e BODDEZ 2016; MucCIOLI 2014 sul rapporto fra culti eroici e culti divini per i sovrani dell'alta età ellenistica. Su specifiche categorie di onorandi, vd. LESCHHORN 1984 (ecisti); FRÖLICH 2013a (membri delle élites civiche); CLAY 2004 e KimMEL-CLAUZET 2013 (poeti). Sugli onori eroici privati, cfr. sotto, par 11.

3. Per l'età ellenistica, ALCOCK 1991 e 1997 mette in relazione l'accresciuta attenzione per i santuari eroici tradizionali con le attività delle élites cittadine e inquadra in questo processo l'uso di definire ň $\rho \omega \varsigma$ la persona onorata. Tali pratiche darebbero forma alla memoria civica e al contempo tramanderebbero, consolidandolo, l'ordine sociale nel quale le élites affermano la propria posizione predominante. Sul contatto tra nuove eroizzazioni e valorizzazione degli eroi mitici in età ellenistica e imperiale, cfr. HUGHES 1999 e JONES 2010. Sulle implicazioni private della relazione fra culto eroico e legame con il territorio, nel contesto delle fondazioni funerarie di età ellenistica e imperiale, vd. CAMPANELLI 2012 e 2017.

4. Con accenti differenti sugli interessi privati (famiglie di proprietari terrieri) o pubblici (ascesa della polis), vd. SNODGRASS 1982, MALKIN 1993 e DE PolignAC 1995, 128-149 sul rapporto fra culto presso la tomba eroica, delimitazione/consacrazione della terra e costruzione di un vincolo di identità in età arcaica. Sulle tendenze del culto eroico in età arcaica e classica, vd. BuRASELIS et al. 2004, 131-151; ЕKROTH 2002 e 2007. Sul culto ecistico in contesto coloniale, LESCHHORN 1984; MALKIN 1987, 189-239; MARI 2012. Sull'apporto dell'iconografia, VouTIRAS 2010 (tipologia dell'eroe a cavallo fra V e IV sec.); FABRICIUS 1999, 2010, 2016 (banchetto funebre, con attenzione particolare per le tendenze ellenistiche). Per una prospettiva archeologica, BOEHRINGER 2001; sugli sviluppi ellenistico-imperiali dell'architettura funeraria monumentale, vd. SCHÖRNER 2007 e VON MANGOLD 
2013; ЕкRотн 2009 offre una sintetica e utile panoramica dei principali indicatori archeologici di un culto eroico.

5. Si vedano tra gli altri ROHDE $1898^{2}$, 356, n. 62; EITREM 1912, coll. 1137-1138; FouCART 1918, 144, n. 51; von WILAMOWITZ - MOELLENDORFF 1932, 19. Secondo questi studiosi, la specializzazione funebre del termine n̆ $\rho \omega \varsigma$ sarebbe segno dell'indebolimento della sua pregnanza cultuale. Tale tesi è seguita da NocK 1944 e 1957, 120-121; LATTIMORE 1942, 97-99; ROBERT 1969, 265; KURTZ - BOARDMAN 1971, 299. Un indizio del precoce "svuotamento" cultuale di funeraria, tenendo conto della particolare diffusione in Beozia, è stato individuato da LATTIMORE in un frammento di Platone comico (Menelaus, fr. 77) nel quale un personaggio si chiede: «Perché non lo impicco, così diventa un eroe a Tebe?». Contra, PIRRotTA 2009, 181-183 suggerisce che il passo alluda a un culto epicorico tebano per un eroe Apenchomenos (cfr. l'epiclesi Apenchomenē per Artemide in Arcadia; Paus. 8, 23, 6).

6. PfuHL - MöBIUS 1977, I 47, e GRAF 1985, 127-131; cfr., recentemente, GRAF 2005, JonES 2010, 53; ЕКROTH 2002 e 2015.

7. ЕKROTH 2015, 386-387.

8. L'impiego retorico e onorifico del vocabolario eroico in età arcaica e classica emerge chiaramente dall'opera di CURRIE 2005. Si vedano anche i contributi di CURRIE e CAMASSA in questo volume.

9. Cfr. par. 5, sui meccanismi di tutela del sepolcro in età imperiale.

10. Attraverso l'analisi della poesia sepolcrale di età ellenistica e imperiale, WYPUSTEK 2013, 65-95 riprende in esame il problema del rapporto fra la presenza della parola ň $\rho \omega \varsigma$ in ambito funerario e la diffusione di forme di immaginario dell'aldilà alternative alla tradizionale rappresentazione della discesa all'Ade. Cfr. sotto, par. 12.

11. I risultati della schedatura (2015-2017) sono pubblicati sul sito di «Epipap, Laboratorio di Epigrafia e Papirologia dell'Università di Padova, Dip. dei Beni Culturali»: (https://http:// www.beniculturali.unipd.it/www/servizi/laboratori/laboratorio-di-epigrafia-antica/ I primi risultati parziali sono stati presentati da A. Coppola a Padova (2014), Milano (2015), Freiburg (2015).

12. Per una presentazione teorica del progetto e delle sue finalità, vd. CANEVA 2020a. Lanciato nel dicembre 2018, il sito del progetto (http://www.phrc.it/) ospita una collezione crescente di iscrizioni relative alla dimensione rituale degli onori per i capi politici e i grandi benefattori del periodo IV-I s. a.C.

13. Vd. recentemente CHRUBASIK - KING 2017 e CHANIOTIS 2018 (IV s. a.C. - II d.C.).

14. Un noto esempio pre-ellenistico è rappresentato dal cosiddetto "Eroe di Temesa" (vicino a Laos, nel Bruzio). La tradizione riportata da Strabone 6.1.5 e Paus. 6.6.4-10 identifica questo eroe con un compagno di Odisseo, chiamato Polite o Alibante, il quale, ucciso dai barbari, avrebbe cominciato a tormentare la popolazione fino a che un oracolo non ingiunse alle popolazioni greche locali di onorarlo come eroe. Un noto esempio di culto epicorico legato al nome "H $\rho \omega \varsigma$ è quello dell'eroe a cavallo attestato in area trace e balcanica, spesso ulteriormente qualificato da epiclesi prettamente locali; lo statuto cultuale di tale figura è tuttavia fluido, dal momento che l'Eroe è spesso chiamato theos (sulla documentazione iconografica e testuale, DiMITROVA 2002).

15. Segnaliamo a margine che in contesti geografici specifici, altre categorie come l'Agathos Daimōn e l'Agathē Tychē di una persona possono essere evocate nel quadro di onori funebri: cfr. la fondazione funeraria di Poseidonios ad Alicarnasso (285-245 a.C.; CGRN 104; LSAM 72; CARBON PirenNe-Delforge 2013), che prevede il culto dell'Agathos Daimōn di Poseidonios e di sua moglie, oltre a quello dell'Agathē Tychē di Poseidonios e dei genitori; CARBON 2005, 5-6 considera il culto del demone personale come tendenza epicorica caria comparabile all'eroizzazione. Sulla tychē personale, cfr. SFAMENI-GASPARRO 1997, 77-78, 89-90.

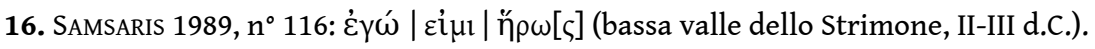


17. IG II ${ }^{2}$ 13193, col. II, linee 1-2 (Attica, c. 150 d.C.). Sulle maledizioni che accompagnano la clausola di inviolabilità nelle fondazioni cultuali e in quelle funerarie (soprattutto in età tardoellenistica e imperiale), vd. PARROT 1939; ROBERT 1978; STRUBBE 1991 (analisi tipologica) e 1997 (catalogo delle fonti epigrafiche microasiatiche); RADUlovA - SuSSU 2015 (area balcanica). Nelle iscrizioni relative alle fondazioni di Antioco I di Commagene (cfr. sotto, nn. 64-66), dopo la clausola d'inviolabilità dei luoghi sacri il sovrano invoca la gratitudine degli dei e degli eroi su coloro che prenderanno buona cura dei luoghi di culto: IGL Syr. I 1 (Nemrud Dağ), linee 186-191. La formula di maledizione è separata dalla clausola di inviolabilità e si distingue dal protocollo comune sia nella forma, sia nella sostituzione degli hērōes con i daimones, la cui ira colpirà $\mathrm{i}$

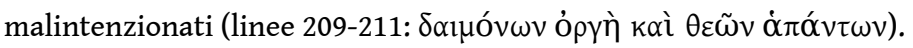

18. IG XII 5, 303 (epigrafe su un sarcofago di Paros, I d.C. ?): il defunto Permenion è definito "il migliore degli eroi"; cfr. Paus. 6.9.7, con citazione dell'oracolo di Delfi che descrive il pugile Kleomedes di Astipalea come "l'ultimo degli eroi".

19. Cfr. I.Aph2007 12.1205 (I/II d.C.), linee 14-15. Sull'istituzione della magistratura eponima perenne, vd. ROBERT 1966, 83-85; GRAF 1985, 127; CHANIOTIS 2004, 407-408; JONES 2010, 71 e 93-94.

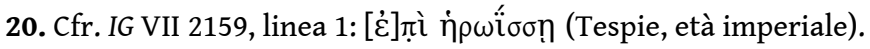

21. L'unica attestazione al dativo di cui siamo a conoscenza nella documentazione epigrafica è integrata in lacuna nel decreto onorifico per Memmios Nikandros a Delfi (125-150 d.C.): FD III 1, 466, linee 8-10. All'accusativo è invece attestata la formula $\dot{\omega} \varsigma \grave{\eta} \rho \omega \tau \iota \mu \tilde{\eta} v$ in un decreto onorifico di Corcira (III a.C.): IG IX 1, 683, linea 8.

22. Tale formula è utilizzata da Erodoto a proposito di Timesios a Teos (1.168), Onesilos a Amatonte (5.114), il persiano Artachaios a Akanthos (7.117). Tucidide la impiega a proposito degli onori per Brasida ad Anfipoli (5.11); Senofonte, Lac. 15.9, per i re spartani. In età imperiale, Pausania ricorre alla stessa formula a proposito degli onori per Aithidas a Messene (4.32.2) e per il pugile Kleomedes di Astipalea (6.9.6-9).

23. Cfr. SEG XLIII 26 A (decreto onorifico del demo di Acharnai, Atene; 315/4 a.C.), linee 3-4.

24. Cfr. SEG XXVIII 1123, iscrizione bilingue da Eumeneia, Frigia (I d.C.).

25. Cfr. IG XII 5, 316 (Paros, I/II d.C.).

26. GRAF 1985, 129, segnala una concentrazione particolare in Ionia e Asia Minore, alle quali in nota 56 è aggiunta la Beozia, già notata da ROHDE 1898², 360, e EITREM 1912, col. 1138; cfr. FRASER 1957, 92-101 e FRASER 1977, 76-81.

27. Il totale delle occorrenze al maschile singolare nella banca dati del PHI è di 1343, di contro alle 178 attestazioni femminili.

28. $I G \mathrm{II}^{2} 1035$ (Attica, I a.C.).

29. IG II ${ }^{2} 839$ (Attica, 221/0 a.C.; vd. GORRINI 2013).

30. Un epigramma di Itanos per tre fratelli eroizzati sottolinea che i tre giovani andranno ad aggiungersi alla lista degli eroi onorati dalla città, a partire da Minosse (IC III iv, 38; Itanos, I a.C.). Un'erma di età imperiale da Cos (Peek GVI 1806; IG XII 4.3, 2925; III d.C.) celebra il poeta tragico Achilleus, eroizzato, indicandolo come figlio di Helpis anziché di Teti. Un epigramma proveniente dalla Troade sottolinea che l'hērōs Mousonios è sepolto nello stesso luogo degli eroi mitici Aiace, Achille e Patroclo (Peek, GVI 1733; IV d.C.). La documentazione di Thera di età imperiale offre varie testimonianze al riguardo: IG XII 3, 869-870 confrontano il dolore per la morte della persona eroizzata con il pianto di Teti per Achille. In IG XII 3, 868, l'omonimia fra l'onorando, Admetos figlio di Theokleides, sacerdote ereditario di Apollo Karneios, e il mitico re di Pherai offre l'occasione di celebrare le origini tessale della famiglia e di confrontare il dolore del padre del defunto con quello di Peleo per Achille.

31. IGUR III 1155 (il poema è attribuito al poeta Marcello di Side). Sull'eroizzazione di Regilla, vd. GLEASON 2010 e JONES 2010, 62.

32. JONES 2010, 67-71. 
33. Si tratta di 484 su 569 occorrenze totali nella banca dati PHI.

34. KUBIŃSKA 1968, 32-57 (sulla dimensione architettonica della tomba); MiKKOLA 2008 (sul profilo storico dell'eroe onorato). Per un approccio giuridico, vd. recentemente ZELNICK-ABRAMOVITZ 2015 sulla proprietà del lotto di terreno su cui è edificata la tomba; RITTI 2004 (Hierapolis), HARTERUipobuU 2010 (Smyrna) e 2014 (Ephesos), HARTER-UibopuU - WIEDERGUT 2014 (Mileto) e RADULOVA 2016 (Tracia e Balcani) sulla regolamentazione dello ius sepulchri nel Mediterraneo orientale di età imperiale. Il diritto romano relativo alla compra-vendita, eredità e protezione delle sepolture costituisce un termine di riferimento fondamentale per comprendere la crescente specializzazione della categoria eroica in relazione alla protezione della tomba; cfr. MANNZMANN 1962; DE VISSCHER 1963; LAZZARINI 1991.

35. Si tratta dei seguenti personaggi: Arato a Sicione; a Sparta, i re Teleklos e Alkon, Kyniska (figlia del re Archidamos II), l'eforo Chilon e infine Athenodoros, un membro della spedizione coloniale in Sicilia del 510 a.C.; a Mantinea Podares, caduto in battaglia contro i Tebani nel 362 a.C. (МiкKоLA 2008, 10, 12-14).

36. Ciò non impedisce che un sepolcro definito come hērōon possa essere venduto, ma il contratto iscritto sul monumento stesso specifica che la compra-vendita ha avuto luogo quando l'edificio era in stato di abbandonato e privo di sepolture: cfr. IKSmyrna 260, con il commento di HARTERUibopuU 2010, 257.

37. Sul rapporto spaziale e funzionale fra la tomba in quanto luogo di sepoltura e il monumento destinato alla commemorazione e al culto del defunto, si veda, per l'età pre-ellenistica, Erodoto 5.47 a proposito del monumento funebre di Filippo di Crotone: in onore del bel Filippo fu costruito un taphos sul quale fu elevato un hērōon destinato a ospitare i sacrifici in suo onore.

38. Cfr. TAM II 55 (Telmesso, età imperiale).

39. Cfr. IKSelge 66 (III d.C.).

40. Cfr. RAmSAY 1895, 335, $n^{\circ} 162$ (Pisidia, età imperiale).

41. Il contenuto di questa sezione riprende in parte la conferenza di S.G. CANEVA, «Acteurs et autorité dans les processus d'héroïsation : à propos des usages du verbe aphērōizzō», Parigi, Collège de France, 27 marzo 2019. Una ricerca compiuta nel TLG (marzo 2019) non ha fornito alcuna attestazione del verbo nelle fonti letterarie. Come si vedrà di seguito, l'impiego esclusivamente documentario del verbo si spiega per la sua stessa natura tecnica, che ne giustifica l'uso in regolamenti rituali e in testi a carattere giuridico.

42. Questa la distribuzione per l'età imperiale: Thera 61 (IG XII 3, 864-877 in documenti pubblici; i documenti privati sono IG XII 3, 893-932, 1266/1635; IG XII 3 Suppl. 1630-1633; IG XII Suppl. 161/699; a questi va aggiunta una iscrizione inedita su altare cilindrico, vista da S.G. Caneva al Museo Archeologico di Thera, settembre 2018); Anaphe 2 (IG XII 3, 281, 288); Chios 1 (BÉ 1974, $n^{\circ}$ 422.18); Atene 1 (IG II ${ }^{2}$ 10531a); Argolide (Trezene) 1 (IG IV 799); Tracia (Perinthos) 1 (McCabe, Perinthos-Herakleia 139); Mesia inferiore (area di Philippopolis) 3 (IG Bulg. III 1, 1005, 1014, 1383); Caria (Aphrodisias) 5 (I.Aph2007 12.320, 322, 524, 526, 909); forse in Frigia (Akmonia) 1 (MAMA VI 312; il verbo è in gran parte integrato in lacuna).

43. Sulla purezza di questi defunti eroizzati, si veda l'uso dell'aggettivo $\dot{\alpha} \gamma v o ́ c ̧$ in IG XII 5, 303, il testo del già citato sarcofago di Paros (I d.c. ?), nel quale si afferma che il tredicenne Parmenion è stato rapito improvvisamente dalla Tychē e condotto nel "luogo puro degli eroi" (sub 4, linee 7-8). La purezza dei defunti eroizzati non deve in ogni caso essere considerata come un aspetto scontato del rapporto greco con gli eroi, come mostrano vari testi da cui si evince un preciso scrupolo relativo ai rischi del contatto con luoghi di culto eroico ai fini del mantenimento di una condizione di purezza rituale: vd. IG XII 4, 332 (CGRN 85), A, linea 14, regolamento da Cos della metà IV sec. a.C., con divieto per un sacerdote di entrare in un hērōon. Tale impiego propriamente cultuale dell'aggettivo $\dot{\alpha} \gamma v o ́ \varsigma$ (o del sinonimo $\kappa \alpha \theta \alpha \rho$ ó $)$ in relazione alla purezza di un eroe o di

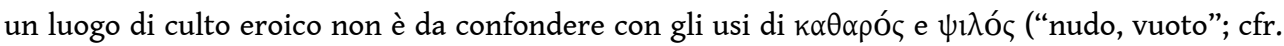


l'italiano "nuda proprietà") in testi sepolcrali di valore giuridico di età imperiale: in questi casi, tali aggettivi corrispondono al latino purus e indicano un lotto di terreno predisposto ad accogliere una tomba, ma non ancora occupato da corpi e perciò ancora privo di vincoli legati allo ius sepulchri (HARTER-UIBOPUU 2010, 255-256).

44. Le ricerche di KUBIŃSKA 1968, 139-147 sulla topografia delle tombe micro-asiatiche offrono alcuni termini di paragone per l'integrazione di un bosco all'interno del terreno di pertinenza di

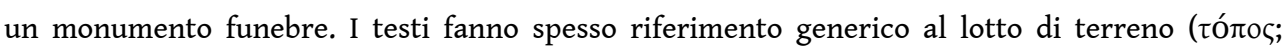
talora ne sono indicate le misure per ragioni di delimitazione della proprietà; CAMPANELLI 2012), oppure al suo perimetro ( $\pi \varepsilon \rho i ́ \beta ం \lambda \circ \varsigma)$, ma non mancano indicazioni più precise relative alla presenza di un giardino ( $\kappa \tilde{\eta} \pi \circ \varsigma)$, di alberi, di un parco ( $\pi \alpha \rho \alpha ́ \delta \varepsilon \varepsilon 1 \sigma o \varsigma)$ o persino di un campo coltivato (’’ypóc). In vari casi il proprietario del monumento esplicita che l'appezzamento di terreno è destinato a fornire agli eredi le rendite che assicureranno lo svolgimento del culto (JHS 19 (1899), 73, n² 25 da Iouliopolis in Galazia). È probabile che la fondazione civica di Itanos per i figli di Ammonios rispondesse alla stessa esigenza insieme cultuale e finanziaria.

45. LAUM 1914, II 63-65, n 50; Hughes 1999, 169; JoNES 2010, 51; sulla dimensione rituale degli onori, cfr. HELMIS 2003, 472-480 ed ЕКRОТн 2015, 391-392; sugli aspetti amministrativi e finanziari della fondazione, HARTER-UIBOPUU 2011, 126-130; SosIN 2014, 57-69. Sulla fondazione di Kritolaos, cfr. par. 10.

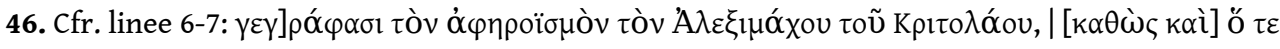
$\delta \tilde{\eta} \mu \circ \varsigma \dot{\varepsilon} \psi \eta \dot{\varphi} \varphi \iota \tau \alpha$ l.

47. Gli onori attribuiti ad Aleximachos comprendono l'erezione di un ritratto del defunto utilizzato come statua cultuale durante le offerte in suo onore - e una festa in occasione della quale si terranno tutte le competizioni previste dalla legge ginnasiarchica di Aigiale per le categorie dei paides e degli andres, a eccezione del pancrazio, il cui vincitore sarà sempre d'ufficio il giovano eroizzato (linee 79-86).

48. LSCG 49; AGRW 21; KLOPPENBORG - AsCOUGH 2011, 179-184, $\mathrm{n}^{\circ} 36$.

49. Cfr. I.Aph2007 12.524, linee 11-18. Sulla terminologia in uso in area micro-asiatica per indicare l'atto di sigillare la tomba (con l'applicazione di grappe di metallo e/o colatura di piombo), KUBIŃSKA 1968, 55-57.

50. IG Bulg. III 1, 1005; IG Bulg. III 1, 1383. Diverso è il caso di IG Bulg. III 1, 1014, appartenente alla stessa tipologia dei documenti privati da Thera e da altre località della Grecia continentale e dell'Egeo, discussi qui di seguito.

51. Sul rapporto fra originale esposto sul monumento e copia archiviata, HARTER-UIPOBUU WIEDERGUT 2014, 160-164.

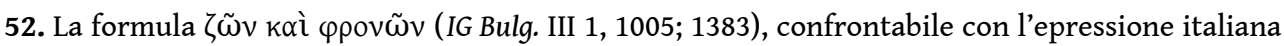
"sano di corpo e di mente", è tipica ed esclusiva del vocabolario testamentario della Tracia/Mesia inferiore di età imperiale. Sulla documentazione funeraria proveniente dai Balcani, RADULOVA SASSU 2015 e RADULOVA 2016.

53. IG II ${ }^{2}$ 974, con le correzioni in SEG XVIII 27.

54. IGR IV 159 (databile probabilmente al regno di Augusto); cfr. linea 12 per il titolo oikistēs, attribuito ad Asklepiades. Su Asklepiades e i suoi discendenti, STRUBBE 2004, 324-325; FERRARY 2005, 62; CHIRICAT 2005, 214-222; FRÖLICH 2013a, 264-266.

55. Per una analisi più dettagliata di questo documento, cfr. sotto, p. 11.

56. IG $\mathrm{II}^{2}$ 1339; K LOPPenborg - Ascough 2011, 217-219, $\mathrm{n}^{\circ} 46$ (AGRW 6). Sulla funzione dell'archieranistēs nelle associazioni private ad Atene, ARNAOUTOGLOU 1994. Sui meccanismi di sottoscrizione e la minaccia di esclusione per i membri morosi, cfr. ARNAOUTOGLOU 2003, 101-102.

57. BCH 12 (1888), 32, $n^{\circ} 11 ;$ McCabe, Mylasa 350.

58. IG XII $1,35$. 
59. IG VII 2725, da Akraipha. Almeno Epaminondas deve essere morto durante l'efebia: alle linee 5-8 si legge che la penale per il mancato rispetto della sacralità del luogo dovrà essere pagata $\tau$ ñ

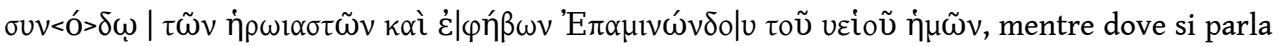
di entrambi i figli, solo gli Hērōistai sono citati. Di particolare interesse, alle linee 22-26,

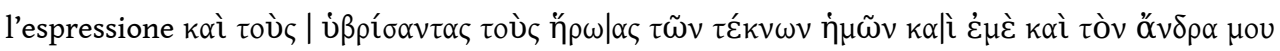
$\Pi v \mid \theta i ́ \omega v \alpha$, dove la distinzione terminologica tra i figli viventi e i figli in quanto eroi permette di identificare i membri defunti della famiglia come persone giuridiche protette dalla clausola di inviolabilità della fondazione.

60. KeIL - VON PREMERSTEIN 1914, 88-90, no. 117; KUBIŃSKA 1968, 125-126.

61. La linea 19 specifica che tale meccanismo di sostituzione in mancanza di prole è valido solo

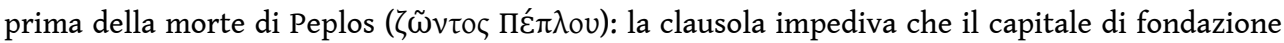
finisse disperso fuori dal ristretto gruppo di familiari e amici di Peplos, una volta che questi, morto, non potesse più esercitare un controllo diretto sull'accesso all'associazione.

62. Per una panoramica della documentazione, oltre al classico ma datato LAUM 1914, 68-74, cfr. Hughes 1999, 168-170; GHERCHANOC 2012, 158-168; HeLMis 2003; JoNES 2010, 50-53; CAMPANELli 2012 e 2017 (da completare con P. HAMON in BÉ 2018, $\mathrm{n}^{\circ}$ 74). Su specifici documenti vd. CARBON - PIRENNEDELFoRge 2013, CAMPANELli 2017 (Diomedon a Cos, CGRN 96, inizio III a.C.; Poseidonios ad Alicarnasso, CGRN 104, c. 285-245 a.C.; Epitteta a Thera, CGRN 152, fine III-inizio II a.C.; su quest'ultimo testo, cfr. WitTENBURG 1990); CAMPANELli 2012 (fondazione testamentaria di Epikrates in Lidia per il figlio Diophantos, I d.C.; HERMANN - PoLATKAN 1969).

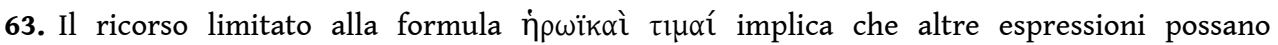
qualificare gli onori per personaggi definiti come eroi: per esempio, in un decreto onorifico di Lykosoura (Arcadia; IG V 2, 517; II/III d.C.), si stabilisce di onorare il defunto eroizzato con la

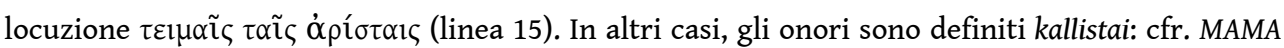
VIII 408 (decreto onorifico per Titus Statilius Apollinarios da Herakleia Salbake, 170 d.C.), linee 15-16. Tali aggettivi non costituiscono di per sé marche di eroizzazione, ma appartengono a una più ampia categoria di onori di massimo grado concessi da una comunità civica a un benefattore.

64. IGL Syr. I 1, linee 206-207 (testo dello hierothesion di Nemrud Dağ; poco prima del 31 a.C.). Sulla cronologia delle iscrizioni dei santuari di Antioco I in Commagene, MITTAG 2011.

65. IGL Syr. I 1, linea 141; IGLSyr. 1, 47, cl. III, linea 26 (testo dello hierothesion di Arsameia sul Nymphaios; 64-38 a.C.).

66. I testi di Nemrud Dağ e di Arsameia sul Nymphaios contengono un altro riferimento agli

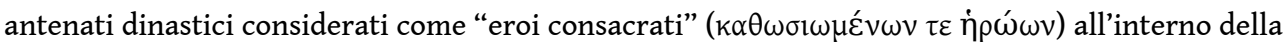
maledizione lanciata contro chi osasse violare i santuari: IGL Syr. I, 1, linea 118 e IGL Syr. I, 47, cl. III, linee 6-7. La stessa formula appare nel testo di più recente pubblicazione proveniente dal santuaro di Ancoz (WAGNER - PETZL 2003, 90-96; SEG LIII 1763, linea 67).

67. Su questo testo, ROBERT 1930, 59-60; BOUSQUET 1966, 443-446. HUGHES 1999, 172 osserva che gli onori per Nikandros non sembrano presentare un contenuto rituale specifico. Che l'impoverimento rituale della categoria eroica costituisca un tratto comune nella documentazione di età imperiale è osservato anche da CAMPANELLI 2012, 77; EKROTH 2015.

68. La formulazione del testo di Polibio non permette di distinguere gli onori concessi dalla Lega Achea da quelli istituiti per iniziativa della madrepatria. È tuttavia probabile che la processione, la festa annuale e l'Arateion sull'agorà menzionati da Plutarco e Pausania costituiscano un'iniziativa specifica della città di Sicione. Sugli onori accordati ad Arato (213/2 c.C.), si vedano, oltre a Polibio 8.12.7-8, Plut., Arat. 53.1-7; sull'hērōon situato sull'agorà, Paus. 2.7.7 e 2.9.4, 6. Per una discussione della documentazione, LESCHHORN 1984, 324-331; GAUTHIER 1985, 60-61; MALKIN 1987, 233-237; SCHÖRNER 2007, 272-274, cat. B16; FRÖLICH 2013a, 250-254; HUGHES 2019. È interessante notare che lo stesso Polibio (12.12b.3) tramanda anche la prima attestazione letteraria della formula i $\sigma o ́ \theta \varepsilon o 1$

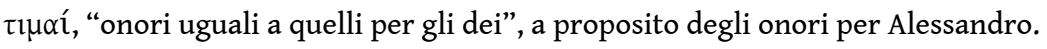


69. Che Diodoro faccia appello a tale categoria al fine di mettere ordine nelle fonti, secondo un obiettivo di sistematizzazione che gli è proprio, emerge chiaramente dai proemi ai libri I e IV, laddove l'autore afferma che dei grandi benefattori dell'età antica, alcuni ricevettero onori/ sacrifici "uguali a quelli per gli dei", altri “eroici” (1.2.4; 4.14). Un'analisi dettagliata dell'impiego delle categorie di ì

70. Sugli onori cultuali per i tiranni in Sicilia, Muccioli 2011.

71. L'unico riferimento a "onori eroici" nei primi libri della Biblioteca concerne Zarinara, regina dei Sakoi, in Asia. Analogamente, in 17.102.4, Diodoro, unico fra le nostre fonti, attribuisce agli Indiani Sambastai l'introduzione di onori eroici per Alessandro (cfr. le osservazioni critiche di GouKowsKI 1976, 258-259).

72. LESCHHORN 1984; MUCCIOLI 2011 e 2014.

73. BURASELIS 2003.

74. Vd. il caso del generale spartano Brasida ad Anfipoli, due secoli prima (422 a.C.): Thuc. 5.11.1, con il commento di FRÖLICH 2013a, 238-239.

75. CANEVA 2020b. Cfr. FRÖLICH 2013b sulle somiglianze fra le cerimonie d'accoglienza regali (apantēseis) di età ellenistica e i contemporanei cortei legati ai funerali pubblici per grandi benefattori.

76. Diod. 29.18; Liv. 39.50.9; CGRN 198 (IG V 2, 432; 183/2 a.C.).

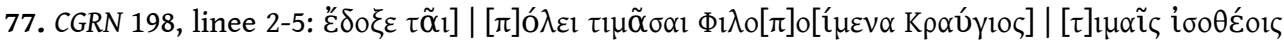

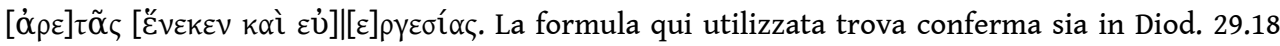
sia in Liv. 39.50.9 (ut ne divinis quidem abstineretur).

78. Su Artemidoro, PALAGIA 1992; TUlly 2014. Sulle sue iniziative cultuali a Thera, GRAF 1995, 107-112. Sull'eroizzazione, BODDEZ 2016, 91.

79. Questa la cronologia più probabile. Contra, TULLY 2014, 201-202, propone una datazione più bassa, durante il regno di Tolemeo VI.

80. IG XII 3, 464, a cui si deve forse aggiungere il frammentario IG XII 3, Suppl. 1388, per il quale le integrazioni di HILLER VON GAERTRINGEN sono tuttavia congetturali.

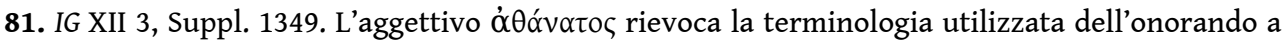
proposito degli altari da lui stesso donati (IG XII 3, 1336; IG XII 3, 1345) e della fama immortale che si era guadagnato in città (IG XII 3,1338). La qualifica di "divino" esalta ulteriormente il carattere ormai sovrumano del suo rapporto con Thera. Sul ricorso all'oracolo di Delfi per il permesso di concedere onori eroici a un benefattore civico, FRöLICH 2013a, 251-252 (Arato a Sicione). È probabile che la moltiplicazione di casi di eroizzazione a livello civico in età ellenistica e imperiale abbia fatto passare in secondo piano la necessità di richiedere un consenso divino per l'istituzione di onori eroici. Tuttavia, la pratica non sembra essere interamente caduta in disuso: IvP III 2 riporta l'interrogazione dell'oracolo di Didima sul migliore luogo in cui i Pergameni avrebbero potuto seppellire gli hērōes Marcellus e Rufinus (Pergamo, II d.C.).

82. Su tale tendenza, oltre al classico GAUTHIER 1985, vd. il recente FORSTER 2018, che offre anche una utile sintesi del dibattito precedente.

83. НАВІсHT 1995, con i riferimenti precedenti.

84. Sulla portata e i limiti dell'evergetismo delle donne delle élites in età ellenistica e imperiale, VAN BREMEN 1996, 25-30; JONES 2008, 199-200.

85. IG XII 7, 447; SÈVE 1996; FRÖLICH 2013a, 289.

86. Tale tendenza non emerge ancora nelle fondazioni più antiche, come quelle di Diomedon a Cos (CGRN 96; inizio III a.C.) e di Poseidonios ad Alicarnasso (CGRN 104; prima metà III a.C.), le quali non presentano tracce di organi deliberativi definiti per statuto (cfr. commento a CGRN 96). La fondazione funeraria del filosofo Epicuro (Diog. Laert., 10.16-20; 270 a.C.) costituisce un caso a se stante, dal momento che la sua gestione è strettamente collegata all'amministrazione della scuola filosofica dopo la morte del fondatore. 
87. Il fondo privato può essere mantenuto attraverso la locazione di proprietà collegate alla fondazione (vd. il caso della fondazione di Diomedon a Cos, CGRN 96, I A 11-17). Per il contributo associativo, cfr. le symbolai istituite dagli Hērōistai in HERMANN - MALAY 2007, n 97, linea 1 (lista di sottoscrittori dell'associazione; la stele accompagna il già citato decreto degli Hērōistai ${ }^{\circ}$ 96, per il quale si segue qui l'edizione di JoNES $2008=$ SEG LVII 1188) e la phora di IG II/III 1339, linea 11 (Hērōistai di Diotimos ad Atene, 57/6 a.C.). I due meccanismi sono probabilmente non in contraddizione l'uno con l'altro: l'attenzione al capitale iniziale si segnala in documenti vicini alla fase di fondazione dell'associazione, mentre i riferimenti a contributi ripetuti o a una quota una tantum di ammissione per i nuovi nati (CGRN 96, I B 51-55) appaiono in testi concernenti la fase di mantenimento delle attività associative. È altresì possibile che in casi eccezionali, i membri dell'associazione potessero fare ricorso a sottoscrizioni occasionali; tale pratica è ben documentata per varie associazioni cultuali private di età ellenistica (MIGEOTTE 2013).

88. AGRW 2; KLOPPENBORG - ASCOUGH 2011, 162-168, nº 33.

89. Sul rapporto tra fondazioni cultuali private e innesto successivo di onori eroici a livello familiare, vd. KAMPS 1937, 151-161 (seguito da SHERWIN-White 1977, 207-217, sui Charmyleoi di Cos) a proposito di una possibile evoluzione da una originaria pratica eroizzante mediata dalla fondazione cultuale, a una procedura di eroizzazione diretta legata alla consacrazione della tomba; MANNZMANN 1962, 136-143, 151-155; recentemente, CAMPANELLI 2012, $71-72$ e il commento al testamento di Epitteta in CGRN 152.

90. GRAF 1985, 127-131.

91. WYPUSTEK 2013; sulla prospettiva di eroizzazione dei defunti e sulla loro auspicata convivenza con gli eroi, gli dei e/o i beati nelle iscrizioni sepolcrali, cfr. LATTIMORE 1942, 31-43, 48-65, 97-106; LEBRIS 2001, 61-120; PERES 2003, 89-121, 196-207, 217-232.

92. Un riesame dettagliato dei casi dubbi, concernenti gli onori per alcuni capi politici di età classica ed ellenistica, sarà offerto da CANEVA, in preparazione.

\section{RIASSUNTI}

L'articolo propone una serie di riflessioni storico-culturali sull'evoluzione dei processi di eroizzazione nel mondo greco durante il periodo ellenistico e imperiale, attraverso il contributo delle fonti scritte (testi epigrafici e letterari). L'analisi lessicale delle occorrenze del termine hērōs e dei suoi derivati, nel tempo e attraverso i differenti generi testuali e supporti mediali, si accompagna a una discussione puntuale dei contesti discorsivi di utilizzo e alla loro dimensione pragmatica, spaziando dalla riflessione sul rapporto fra vecchi e nuovi eroi alla definizione degli aspetti che rendono una tomba 'eroica' sotto il profilo legale, dalla costruzione di un immaginario eroico dell'aldilà alle implicazioni socio-economiche dell'istituzione e amministrazione di onori cultuali per i defunti, nella sfera pubblica come nel contesto privato di associazioni familiari. L'ampio spettro delle sfere di applicazione delle parole connesse alla sfera semantica dell'hērōs conferma la piena vitalità di questo aspetto della religione greca postclassica, inducendoci a parlare non tanto di declino o di metaforizzazione della categoria eroica, ma di un ampliamento dei suoi contesti sociali e culturali di utilizzo. Tale tendenza inevitabilmente comportò una serie di adattamenti della sfera semantica di hērōs alle nuove esigenze e aspettative degli attori coinvolti, senza tuttavia privare la parola della sua duratura rilevanza religiosa. 
This paper deals with the evolution of heroic cults and honours in the Greek world in the Hellenistic and Imperial periods from the point of view of textual sources (epigraphic and literary texts). The discussion of the occurrences of the word hērōs and of its related terms, which aims at identifying variations across time and media, combines with an attentive analysis of the discursive and pragmatic contexts where these words are used. The scope of this contribution therefore encompasses topics such as the perception of the relationship between old and new heroes, the legal features characterizing a tomb as 'heroic', the definition of the imaginaire of a heroic afterlife, and the socio-economic implications of the institution and administration of cultic honours for the dead, in the public sphere as well as in private family associations. The broad spectrum of use which concerns the words connected with the semantic sphere of herōs confirms the vitality of this aspect of post-classical Greek religion, compellingly suggesting that we should replace the idea of decline and metaphorization of the category of hero with that of the broadening of the social and cultural contexts of its application. This tendency of course entailed a series of adaptations of the semantic sphere of hērōs to the new needs and agendas of the involved agents, without, however, depriving this word of its long-lasting religious significance.

\section{INDICE}

Keywords : heroic honours, textual evidence, old/new heroes, cultic associations, tomb and afterlife

Parole chiave : onori eroici, fonti testuali, vecchi/nuovi eroi, associazioni cultuali, tomba e aldilà

\section{AUTORI}

\section{STEFANO G. CANEVA}

Università degli Studi di Padova

Dipartimento dei Beni Culturali

Piazza Capitaniato, 7

35139 Padova, Italia

stefano.caneva(at)unipd.it

ALESSANDRA COPPOLA

Università degli Studi di Padova

Dipartimento dei Beni Culturali

Piazza Capitaniato, 7

35139 Padova, Italia

alessandra.coppola(at)unipd.it 